\title{
Existence and large-time asymptotics for solutions of semilinear parabolic systems with measure data
}

\author{
TOMASZ KLIMSIAK
}

Abstract. We study the Cauchy-Dirichlet problem for monotone semilinear uniformly elliptic second-order parabolic systems in divergence form with measure data. We show that under mild integrability conditions on the data, there exists a unique probabilistic solution of the system. We also show that if the operator and the data do not depend on time, then the solution of the parabolic system converges as $t \rightarrow \infty$ to the solution of the Dirichlet problem for an associated elliptic system. In fact, we prove some results on the rate of the convergence.

\section{Introduction}

Let $D \subset \mathbb{R}^{d}, d \geq 2$ be an open bounded domain. In the present paper, we study systems of the form

$$
\left\{\begin{array}{l}
\frac{\partial u^{k}}{\partial t}-A_{t} u^{k}=f^{k}(t, x, u)+\mu^{k} \quad \text { in } D_{T}, \quad k=1, \ldots, N, \\
u_{\mid \partial D}(t, \cdot)=0, t \in(0, T], \quad u(0, \cdot)=\varphi \quad \text { on } D .
\end{array}\right.
$$

Here, $D_{T} \equiv[0, T] \times D, \mu^{k}, k=1, \ldots, N$ are bounded soft measures on $\mathbb{R}_{+} \times D$, i.e., bounded Borel measures absolutely continuous with respect to the parabolic capacity determined by the operator

$$
A_{t}=\frac{1}{2} \sum_{i, j=1}^{d} \frac{\partial}{\partial x_{j}}\left(a_{i j}(t, x) \frac{\partial}{\partial x_{i}}\right) .
$$

In this paper, we assume that $f: D_{T} \times \mathbb{R}^{N} \rightarrow \mathbb{R}^{N}$ is a continuous monotone vector field, i.e., $f(t, x, \cdot)$ is continuous for a.e. $(t, x) \in D_{T}$ and for some $\alpha \in \mathbb{R}$,

$$
\left\langle f(t, x, y)-f\left(t, x, y^{\prime}\right), y-y^{\prime}\right\rangle \leq \alpha\left|y-y^{\prime}\right|^{2}
$$

for a.e. $(t, x) \in D_{T}$ and every $y, y^{\prime} \in \mathbb{R}^{N}$. Concerning the growth of $f$, we merely require $f$ to satisfy the following condition

$$
f(\cdot, \cdot, 0) \in L^{1}\left(D_{T}\right), \quad \forall_{r>0, y \in \mathbb{R}^{N}} R^{0, T}\left(\sup _{|y| \leq r}|f(\cdot, \cdot, y)|\right)<\infty, \quad m_{1} \text {-a.s. },
$$

Mathematics Subject Classification (2010): Primary 35J91, 35J57; Secondary 60H30

Keywords: Semilinear parabolic system, Divergence form operator, Measure data, Large-time behavior, Backward stochastic differential equation. 
where $R^{0, T}$ is the potential operator of $\frac{\partial}{\partial t}-A_{t}$ on $D_{T}$. As for $A_{t}$, we assume that its coefficient $a: D_{T} \rightarrow \mathbb{R}^{d} \otimes \mathbb{R}^{d}$ is a measurable symmetric matrix-valued function such that for some $\Lambda \geq 1$,

$$
\Lambda^{-1}|\xi|^{2} \leq \sum_{i j=1}^{d} a_{i j}(t, x) \xi_{i} \xi_{j} \leq \Lambda|\xi|^{2}, \quad \xi \in \mathbb{R}^{d}
$$

for a.e. $(t, x) \in D_{T}$.

The aim of the present paper was twofold. In the first part of the paper, we focus on the problem of existence of solutions to (1.1). One of the possible approaches to the problem (1.1) is to use the framework of renormalized (or entropy) solutions. Such a framework was successfully applied to scalar equations of the type (1.1) with Leray-Lions-type operator $A_{t}$ (see, e.g., [2]). For a function $u$ on $D_{T}$, put

$$
f_{u}(t, x)=f(t, x, u(t, x)), \quad(t, x) \in D_{T} .
$$

We say that $u: D_{T} \rightarrow \mathbb{R}^{k}$ is a renormalized solution of (1.1) if $f_{u} \in L^{1}\left(D_{T}\right), u$ belongs to the space $\mathcal{T}_{2}^{0,1}$, i.e., for every $s \geq 0, T_{s}\left(u^{k}\right)=\left((-s) \vee u^{k}\right) \wedge s \in W_{2}^{0,1}\left(D_{T}\right)$ for $k=1, \ldots, N$, and if $u^{k}, k=1, \ldots, N$ is a renormalized solution of the linear Cauchy-Dirichlet problem

$$
\frac{\partial u^{k}}{\partial t}-A_{t} u^{k}=f_{u}^{k}(t, x)+\mu^{k}, \quad u^{k}(t, \cdot)_{\mid \partial D}=0, \quad t \in(0, T], \quad u(0, \cdot)=\varphi .
$$

Unfortunately, showing that $f_{u} \in L^{1}\left(D_{T}\right)$ under the growth condition (1.4) is in general a complicated, if ever possible, task. Consequently, we do not know whether $u \in \mathcal{T}_{2}^{0,1}$, and the concept of renormalized solutions is not applicable. However, we are able to prove that under (1.4), the function $f_{u}$ is quasi-integrable, i.e., roughly speaking, integrable except possibly on sets of small capacity (see Sect. 5 for details). This in turn implies that $u$ belongs to the stochastic Sobolev space $W^{0,1}\left(\mathbb{X}^{D_{T}}\right)$ introduced in [14] to investigate the obstacle problem with possible nowhere Radon reflection measure. The space $W^{0,1}\left(\mathbb{X}^{D_{T}}\right)$ is wider than $\mathcal{T}_{2}^{0,1}$ but their elements are regular enough to define correctly probabilistic solutions of $(1.1)$ in $W^{0,1}\left(\mathbb{X}^{D_{T}}\right)$ and prove the uniqueness result.

For $u \in \mathcal{B}\left(D_{T}\right)$, let $\bar{u}(t, x)=u(T-t, x)$, and for a bounded soft measure $\mu$ on $D_{T}$, let $\bar{\mu}$ be the bounded soft measure on $D_{T}$ such that $\int \bar{\eta} \mathrm{d} \mu=\int \eta \mathrm{d} \bar{\mu}$ for every $\eta \in \mathcal{B}_{b}\left(D_{T}\right)$. Roughly speaking, $u \in W^{0,1}\left(\mathbb{X}^{D_{T}}\right)$ is a probabilistic solution of (1.1) if for quasi-every (q.e. for short) $(s, x) \in D_{T}$,

$$
\begin{aligned}
\bar{u}\left(t, X_{t}\right)= & \mathbf{1}_{\left\{\zeta^{s}>T\right\}} \varphi\left(X_{T}\right)+\int_{t}^{T \wedge \zeta^{s}} \bar{f}_{u}\left(r, X_{r}\right) \mathrm{d} r+\int_{t}^{T \wedge \zeta^{s}} \mathrm{~d} A_{r}^{\bar{\mu}} \\
& -\int_{t}^{T \wedge \zeta^{s}} \bar{\sigma} \nabla_{\mathbb{X}} \bar{u}\left(r, X_{r}\right) \mathrm{d} B_{r}, \quad t \in\left[0, T \wedge \zeta^{s}\right], \quad P_{s, x} \text {-a.s. },
\end{aligned}
$$


where $\sigma \cdot \sigma^{T}=a, \mathbb{X}=\left\{\left(X, P_{s, x}\right) ;(s, x) \in \mathbb{R}_{+} \times D\right\}$ is a time-inhomogeneous Markov process associated with the operator $A_{t}, \zeta^{s}$ is the first exit time of $\left(X, P_{s, x}\right)$ from $D$, i.e.,

$$
\zeta^{s}=\inf \left\{t \geq s: X_{t} \notin D\right\},
$$

and $A^{\mu}$ is the additive functional of $\mathbb{X}$ in the Revuz correspondence with $\mu$. In the last integral in (1.6), $B$ is a standard $d$-dimensional Brownian motion and $\nabla_{\mathbb{X}} \bar{u}$ stands for the stochastic gradient of $\bar{u}$ (see [14] or Sect. 5). Formula (1.6) can be regarded as a nonlinear Feynman-Kac formula, because taking $t=s$ and integrating it with respect to the measure $P_{s, x}$, we get

$$
\bar{u}(s, x)=E_{s, x}\left(\mathbf{1}_{\left\{\zeta^{s}>T\right\}} \varphi\left(X_{T}\right)+\int_{s}^{T \wedge \zeta^{s}} \bar{f}_{u}\left(r, X_{r}\right) \mathrm{d} r+\int_{s}^{T \wedge \zeta^{s}} \mathrm{~d} A_{r}^{\bar{\mu}}\right)
$$

whenever the above integrals exist. We would like to stress that our probabilistic solution $u$ to (1.1) may be considered as some generalization of the notion of renormalized (or entropy) solution, because if $f_{u} \in L^{1}\left(D_{T}\right)$, then $u \in \mathcal{T}_{2}^{0,1}, u \in$ $L^{q}\left(0, T ; W_{0}^{1, q}(D)\right)$ for $q \in\left[1, \frac{d+2}{d+1}\right)$ and $u$ is a renormalized (and entropy as well) solution to (1.1) (see Remark 5.14). Perhaps, also the following comment is appropriate at this point, although the probabilistic solution of (1.1) is in general weak and at first glance its definition seems complicated, it is actually very convenient to deal with.

Our results on the existence and uniqueness of solutions of (1.1) generalize known results in the sense that we consider semilinear parabolic systems with measure data (semilinear elliptic systems with measure data are considered in [15,23]). We should also stress that our results are proved for systems with $f$ satisfying quite general condition (1.3) for which the usual monotonicity methods do not apply and we only require $f$ to satisfy mild integrability condition (1.4) analogous to the integrability condition considered for elliptic equations or systems in $[2,15,23]$. We also allow $f$ to depend on $x$.

In the second part of the paper, we investigate the asymptotic behavior as $t \rightarrow \infty$ of probabilistic solutions of (1.1) in the case where $A_{t}=A, f$ and $\mu=\left(\mu^{1}, \ldots, \mu^{N}\right)$ do not depend on time. Let $\tilde{\mu}^{k}(B)=\mu^{k}([0,1] \times B), k=1, \ldots, N$, for any Borel subset $B$ of $D$. Our main result says that under the assumptions ensuring the existence and uniqueness of a solution $v$ to the elliptic system

$$
\left\{\begin{array}{l}
-A v^{k}=f^{k}(x, v)+\tilde{\mu}^{k} \quad \text { in } D, \quad k=1, \ldots, N \\
v_{\mid \partial D}=0
\end{array}\right.
$$

we have

$$
u(t, x) \rightarrow v(x) \text { as } t \rightarrow \infty
$$

for q.e. $x \in D$. As a matter of fact, we prove that there is $c$ depending only on $d, \Lambda$ such that for every $t>0$ and $q \in(0,1)$, 


$$
\begin{aligned}
|u(t, x)-v(x)| \leq & c(1-q)^{-1 / q} t^{-d / 2}\left(\left(P_{x}\left(\zeta^{0}>t\right)\right)^{(1-q) / q}\|\varphi\|_{L^{1}}\right. \\
& \left.+\|R f(\cdot, 0)\|_{L^{1}}+\|R \tilde{\mu}\|_{L^{1}} \leq c(1-q)^{-1 / q} t^{-d / 2}\right)
\end{aligned}
$$

for q.e. $x \in D$, where $R$ is the potential operator of $-A$ on $D$. From this, it follows in particular that there exists $c=c(\Lambda, d,|D|)$ such that for every $t>0$,

$$
|u(t, x)-v(x)| \leq c(1-q)^{-1 / q} t^{-d / 2}\left(\mathrm{e}^{-b t(1-q) / q}\|\varphi\|_{L^{1}}+\|f(\cdot, 0)\|_{L^{1}}+\|\tilde{\mu}\|_{T V}\right),
$$

because it is known that $P_{x}\left(\zeta^{0}>t\right) \leq a \mathrm{e}^{-b t}$ for some $a, b>0$ depending only on $d, \Lambda$ and $|D|$ (the Lebesgue measure of $D$ ) and $\|R \tilde{\mu}\|_{L^{1}} \leq C(d, \lambda,|D|)\|\tilde{\mu}\|_{T V}\left(\|\tilde{\mu}\|_{T V}\right.$ stands for the total variation of $\tilde{\mu})$. For instance, if $f(\cdot, 0)=0$ and $\mu(\mathrm{d} x)=0$, then the rate of convergence in (1.11) is the same as in the classical case of one linear equation (see [8]). We also show that in fact (1.10) holds for every $x$ from the set

$$
F_{0}=\left\{x \in D ; \forall r>0 R|f(\cdot, 0)|(x)+(R \tilde{\mu})(x)+R\left(\sup _{|y| \leq r}|f(\cdot, y)|\right)(x)<\infty\right\} .
$$

In case $N=1$, the large-time asymptotic behavior of solutions to parabolic equations with measure or $L^{1}$ data was investigated in [21,27-29] (in [27], the case of general, possibly singular measures is considered). In all these papers in proofs, some comparison results are used. Therefore, the methods of [21,27-29] cannot be applied to systems considered in the present paper. Let us also point out that these methods do not provide estimates of the difference between solutions to parabolic equations and the corresponding stationary solutions.

Although the main results of the paper concern systems of PDEs and are analytic in nature, the methods of proofs are those of stochastic analysis, Markov processes, and especially the theory of backward stochastic differential equations. Therefore, in Sects. 2-4, we give relevant background material concerning these topics. Then, in Sect. 5, we prove our results on the existence and uniqueness of solutions of (1.1), and in Sect. 6 results on their large-time behavior. Our idea of using the methods of backward stochastic differential equations to the study of large-time behavior of semilinear parabolic equations is new. It seems likely that it can be applied to wider that (1.1) class of equations.

\section{Preliminary results}

Let us fix a probability space $(\Omega, \mathcal{F}, P)$ equipped with a filtration $\left\{\mathcal{F}_{t}\right\}$ satisfying the usual conditions.

By $B$, we denote a standard $d$-dimensional $\left\{\mathcal{F}_{t}\right\}$-Brownian motion. By $\mathcal{A}$, we denote the set of all $\left\{\mathcal{F}_{t}\right\}$ progressively measurable real-valued processes and by $\mathcal{V}$ (respectively, $\mathcal{V}_{c}$ ) the subspace of $\mathcal{A}$ consisting of all increasing càdlàg (respectively, continuous) processes $Y$ such that $Y_{0}=0 . M$ is the space of all processes $Z \in \mathcal{A}$ such that $P\left(\int_{0}^{T}\left|Z_{t}\right|^{2} \mathrm{~d} t<\infty\right)=1$ for every $T>0 . M^{p}, p>0$, is the subspace of 
$M$ consisting of all processes such that $E\left(\int_{0}^{\infty}\left|Z_{r}\right|^{2} \mathrm{~d} r\right)^{p / 2}<\infty$. By $\mathcal{D}$ (respectively, $\mathcal{S}$ ), we denote the space of all càdlàg (respectively, continuous) processes in $\mathcal{A}$, and by $\mathcal{D}^{p}$ (respectively, $\mathcal{S}^{p}$ ), $p>0$, the space of all processes $Y \in \mathcal{D}$ (respectively, $Y \in \mathcal{S}$ ) such that $E \sup _{t \geq 0}\left|Y_{t}\right|^{p}<\infty$. We say that a process $Y$ is of class (D) if $Y \in \mathcal{A}$ and the family $\left\{Y_{\tau}, \tau \in \mathcal{T}\right\}$, where $\mathcal{T}$ is the set of all finite $\left\{\mathcal{F}_{t}\right\}$-stopping times, is uniformly integrable. For a càdlàg process $Y$, we write

$$
\Delta Y_{t}=Y_{t}-Y_{t-}, \quad Y_{t-}=\lim _{s \nearrow t} Y_{s}
$$

In the paper, we adopt the following convention. If $S$ is a space of real functions and $N, d \in \mathbb{N}$, then by $[S]^{N}$ (respectively, $[S]^{N \times d}$ ), we denote the space of all functions of the form $f=\left(f^{1}, \ldots, f^{N}\right)$ (respectively, $\left.f=\left[f^{i, j}\right]_{N \times d}\right)$ such that $f^{i} \in S$ for $i=1, \ldots, N$ (respectively, $f^{i, j} \in S$ for $i=1, \ldots, N, j=1, \ldots, d$ ). If $A$ is an $N \times d$-dimensional real matrix, then $|A|$ stands for trace $A A^{*}$.

Write

$$
\hat{x}=\operatorname{sgn}(x)=\mathbf{1}_{\{x \neq 0\}} \frac{x}{|x|}, \quad x \in \mathbb{R}^{N} .
$$

The following multidimensional version of the Itô-Tanaka formula will be frequently used in the paper.

PROPOSITION 2.1. Let X be a progressively measurable process such that

$$
X_{t}=X_{0}+\int_{0}^{t} \mathrm{~d} K_{s}+\int_{0}^{t} H_{s} \mathrm{~d} B_{s}, \quad t \geq 0
$$

for some $K \in[\mathcal{V}]^{N}, H \in[M]^{N \times d}$. Then, there is $L \in \mathcal{V}$ such that for every $p \geq 1$,

$$
\begin{aligned}
\left|X_{t}\right|^{p}= & \left|X_{0}\right|^{p}+p \int_{0}^{t}\left|X_{s-}\right|^{p-1}\left\langle\hat{X}_{s-}, \mathrm{d} K_{s}\right\rangle+p \int_{0}^{t}\left|X_{s}\right|^{p-1}\left\langle\hat{X}_{s}, H_{s} \mathrm{~d} B_{s}\right\rangle \\
& +\frac{p}{2} \int_{0}^{t}\left|X_{S}\right|^{p-2} \mathbf{1}_{X_{s \neq 0}}\left\{(2-p)\left(\left|H_{s}\right|^{2}-\left\langle\hat{X}_{s}, H_{s} H_{s}^{*} \hat{X}_{s}\right\rangle\right)+(p-1)\left|H_{s}\right|^{2}\right\} \mathrm{d} s \\
& +\mathbf{1}_{\{p=1\}} L_{t}+\sum_{0<s \leq t}\left(\Delta\left|X_{S}\right|^{p}-\left\langle\left|X_{S}\right|^{p-1} \hat{X}_{s}, \Delta X_{s}\right\rangle\right), \quad t \geq 0 .
\end{aligned}
$$

Proof. The proof is a modification of the proof of [3, Lemma 2.2]. Obviously, it suffices to prove the formula for $t \in[0, T]$. Set $u_{\varepsilon}(x)=\left(|x|^{2}+\varepsilon^{2}\right)^{1 / 2}, x \in \mathbb{R}^{N}, \varepsilon>0$. A straightforward computation shows that

$$
\nabla u_{\varepsilon}^{p}(x)=p u_{\varepsilon}^{p-2}(x) x, D^{2} u_{\varepsilon}^{p}(x)=p u_{\varepsilon}^{p-2}(x) I+p(p-2) u_{\varepsilon}^{p-4}(x)(x \otimes x),
$$

where $I$ is the $n$-dimensional identity matrix. By the Itô-Meyer formula, 


$$
\begin{aligned}
u_{\varepsilon}^{p}\left(X_{S}\right)= & u_{\varepsilon}^{p}\left(X_{0}\right)+\int_{0}^{t}\left\langle\nabla u_{\varepsilon}^{p}\left(X_{s-}\right), \mathrm{d} K_{s}\right\rangle+\int_{0}^{t}\left\langle\nabla u_{\varepsilon}^{p}\left(X_{S-}\right), H_{S} \mathrm{~d} B_{S}\right\rangle \\
& +\frac{1}{2} \sum_{i, j} \int_{0}^{t} \frac{\partial^{2} u_{\varepsilon}^{p}}{\partial x_{i} \partial x_{j}}\left(X_{s-}\right) d\left[X^{i}, X^{j}\right]_{s}^{c}+\sum_{0<s \leq t}\left(\Delta u_{\varepsilon}^{p}\left(X_{S}\right)-\left\langle\nabla u_{\varepsilon}^{p}\left(X_{s-}\right), \Delta X_{S}\right\rangle\right) \\
= & u_{\varepsilon}^{p}\left(X_{0}\right)+\sum_{i=1}^{4} I_{i}^{\varepsilon}(t) .
\end{aligned}
$$

It is clear that

$$
u_{\varepsilon}^{p}\left(X_{t}\right) \rightarrow\left|X_{t}\right|^{p}, \quad I_{1}^{\varepsilon}(t) \rightarrow p \int_{0}^{t}\left|X_{s-}\right|^{p-1}\left\langle\hat{X}_{s-}, \mathrm{d} K_{s}\right\rangle, \quad \varepsilon \searrow 0
$$

a.s. for $t \in[0, T]$. Using arguments from the proof of [3, Lemma 2.2], one can show that

$$
I_{2}^{\varepsilon}(t) \rightarrow p \int_{0}^{t}\left|X_{s}\right|^{p-1}\left\langle\hat{X}_{s}, H_{S} \mathrm{~d} B_{s}\right\rangle, \quad \varepsilon \searrow 0
$$

uniformly on $[0, T]$ in probability, and that $I_{3}^{\varepsilon}(t)=I_{3,1}^{\varepsilon}(t)+I_{3,2}^{\varepsilon}(t)$, where

$$
\begin{aligned}
I_{3,1}^{\varepsilon}(t)= & \frac{p(p-2)}{2} \int_{0}^{t}\left(\left|X_{S}\right| u_{\varepsilon}^{-1}\left(X_{S}\right)\right)^{4-p}\left|X_{S}\right|^{p-2} \mathbf{1}_{\left\{X_{s} \neq 0\right\}}\left(\left|H_{S}\right|^{2}-\left\langle\hat{X}_{s}, H_{S} H_{S}^{*} \hat{X}_{s}\right\rangle\right) \mathrm{d} s \\
& +p(p-1)\left(\left|X_{S}\right| u_{\varepsilon}^{-1}\left(X_{S}\right)\right)^{4-p}\left|X_{S}\right|^{p-2} \mathbf{1}_{\left\{X_{S} \neq 0\right\}}\left|H_{S}\right|^{2}
\end{aligned}
$$

and

$$
I_{3,2}^{\varepsilon}(t)=\varepsilon^{2} \frac{p}{2} \int_{0}^{t}\left|H_{S}\right|^{2} u_{\varepsilon}^{p-4}\left(X_{S}\right) \mathrm{d} s
$$

Moreover, as $\varepsilon \searrow 0$,

$$
I_{3,1}^{\varepsilon}(t) \rightarrow \int_{0}^{t}\left|X_{S}\right|^{p-1} \mathbf{1}_{\left\{X_{s} \neq 0\right\}}\left\{(2-p)\left(\left|H_{S}\right|^{2}-\left\langle\hat{X}_{s}, H_{s} H_{s}^{*} \hat{X}_{s}\right\rangle\right)-(p-1)\left|H_{S}\right|^{2}\right\} \mathrm{d} s
$$

a.s. for every $t \in[0, T]$. We now show the convergence of $I_{4}^{\varepsilon}(t)$. It is clear that

$$
\Delta u_{\varepsilon}^{p}\left(X_{s}\right) \rightarrow \Delta\left|X_{s}\right|^{p}, \quad\left\langle\nabla u_{\varepsilon}^{p}\left(X_{s-}\right), \Delta X_{s}\right\rangle \rightarrow\left\langle\left|X_{S}\right|^{p-1} \hat{X}_{s}, \Delta X_{s}\right\rangle, \quad s \in[0, T] .
$$

Observe also that

$$
\left|\Delta u_{\varepsilon}^{p}\left(X_{s}\right)\right| \leq \sup _{\theta \in[0,1]}\left|\nabla u_{\varepsilon}^{p}\left(\theta \Delta X_{s}+X_{s-}\right)\right|\left|\Delta X_{s}\right| \leq 3 \sup _{0 \leq t \leq T}\left(\left|X_{t}\right|^{p-1}+1\right)\left|\Delta X_{s}\right|
$$

and

$$
\left|\nabla u_{\varepsilon}^{p}\left(X_{s-}\right)\right| \leq \sup _{0 \leq t \leq T}\left(\left|X_{t}\right|^{p-1}+1\right)
$$


Since $\sum_{0<t<T}\left|\Delta X_{S}\right| \leq\left|K_{T}\right|$, applying the Lebesgue dominated convergence theorem shows that for every $t \in[0, T]$,

$$
I_{4}^{\varepsilon}(t) \rightarrow \sum_{0<s \leq t}\left(\Delta\left|X_{s}\right|^{p}-\left\langle\left|X_{s}\right|^{p-1} \hat{X}_{s}, \Delta X_{s}\right\rangle\right), \quad \varepsilon \searrow 0 .
$$

By what has already been proved, it follows that $I_{3,2}^{\varepsilon}(t)$ is convergent. Put $L_{t}(p)=$ $\lim _{\varepsilon \rightarrow 0} I_{3,2}^{\varepsilon}(t)$. Then, $L$ is a càdlàg increasing process, and as in the proof $[3$, Lemma 2.2], one can show that if $p>1$, then $L_{t}(p)=0$ for $t \in[0, T]$, which completes the proof.

REMARK 2.2. It is well known that the function $v(x)=p|x|^{p-1} \hat{x}$ is the subgradient of the function $u(x)=|x|^{p}$. Therefore, $u(x)-u(y) \geq\langle x-y, v(x)\rangle, x, y \in \mathbb{R}^{N}$. This implies that the process

$$
I_{t}=\sum_{0<s \leq t}\left(\Delta\left|X_{s}\right|^{p}-\left\langle\left|X_{s}\right|^{p-1} \hat{X}_{s}, \Delta X_{s}\right\rangle\right), \quad t \geq 0
$$

is increasing.

COROLLARY 2.3. Under the assumptions of Proposition 2.1, for every $0 \leq t \leq T$ and $p \geq 1$,

$$
\begin{aligned}
\left|X_{t}\right|^{p}+c(p) \int_{t}^{T}\left|X_{s}\right|^{p-2} \mathbf{1}_{\left\{X_{s} \neq 0\right\}}\left|H_{s}\right|^{p} \mathrm{~d} s & \leq\left|X_{T}\right|^{p}-p \int_{t}^{T}\left|X_{s-}\right|^{p-1}\left\langle\hat{X}_{s-}, \mathrm{d} K_{s}\right\rangle \\
& -\int_{t}^{T}\left|X_{S}\right|^{p-1}\left\langle\hat{X}_{s}, H_{s} \mathrm{~d} B_{S}\right\rangle,
\end{aligned}
$$

where $c(p)=p[(p-1) \wedge 1] / 2$.

\section{Backward stochastic differential equations}

Let $B$ denote a standard $d$-dimensional $\left\{\mathcal{F}_{t}\right\}$-Brownian motion. Let $\sigma$ be a bounded $\left\{\mathcal{F}_{t}\right\}$-stopping time, $\xi$ be an $\mathcal{F}_{\sigma}$-measurable random variable, $A \in \mathcal{V}$ and let $f$ : $\Omega \times \mathbb{R}_{+} \times \mathbb{R}^{N} \rightarrow \mathbb{R}^{N}$ be a measurable function such that $f(\cdot, y)$ is progressively measurable for every $y \in \mathbb{R}^{N}$.

Let us recall that a pair $(Y, Z)$ consisting of an $\mathbb{R}^{N}$-valued process $Y$ and an $\mathbb{R}^{N \times d_{\text {- }}}$ valued processes $Z$ is called a solution of $\operatorname{BSDE}(\xi, \sigma, f+\mathrm{d} A)$ if

(a) $Y, Z$ are $\left\{\mathcal{F}_{t}\right\}$-progressively measurable, $Y$ is càdlàg, $P\left(\int_{0}^{\sigma}\left|Z_{t}\right|^{2} \mathrm{~d} t<\infty\right)=1$,

(b) $t \mapsto f\left(t, Y_{t}\right) \in L^{1}(0, \sigma), P$-a.s.,

(c) $Y_{t}=\xi+\int_{t}^{\sigma} f\left(s, Y_{s}\right) \mathrm{d} s+\int_{t}^{\sigma} \mathrm{d} A_{s}-\int_{t}^{\sigma} Z_{s} \mathrm{~d} B_{s}, 0 \leq t \leq \sigma, P$-a.s.

Let $(Y, Z)$ be a solution of $\operatorname{BSDE}(\xi, \sigma, f+\mathrm{d} A)$. By putting $Y_{t}=\xi, t \geq \sigma, Z_{t}=$ $0, t \geq \sigma$, we may and will assume in the sequel that the processes $Y, Z$ are defined for $t \geq 0$. We also adopt the convention that $\int_{a}^{b}=0$ for $a \geq b$. Then, the stochastic equation in (c) is satisfied for every $t \geq 0$.

Let us consider the following assumptions. 
(A1) $E\left(|\xi|^{p}+\left(\int_{0}^{\sigma}|f(t, 0)| \mathrm{d} t\right)^{p}+|A|_{\sigma}^{p}\right)<+\infty$.

(A2) There is $\mu \in \mathbb{R}$ such that $\left\langle y-y^{\prime}, f(t, y)-f\left(t, y^{\prime}\right)\right\rangle \leq \mu\left|y-y^{\prime}\right|^{2}$ for every $t \geq 0, y, y^{\prime} \in \mathbb{R}^{N}$.

(A3) For every $t \geq 0, y \mapsto f(t, y)$ is continuous.

(A4) For every $r>0, E \int_{0}^{\sigma} \sup _{|y| \leq r}|f(t, y)| \mathrm{d} t<\infty$.

In [3, Theorem 4.2], it is proved that under (A1)-(A4) with $p>1$, there exists a unique solution $(Y, Z) \in \mathcal{S}^{p} \otimes M^{p}$ of $\operatorname{BSDE}(\xi, \sigma, f)$. We will show how to modify the proof of [3, Theorem 4.2] to get the existence and uniqueness in the general case, i.e., for $p \geq 1$ and nonzero process $A$.

The proof of [3, Theorem 4.2] is based on Lemma 3.1 and Proposition 3.2 in [3], [26, Theorem 2.2] and [4, Lemma 2.2]. To state the generalizations of Lemma 3.1 and Proposition 3.2 in [3] to equations with nonzero $\mathrm{d} A$, we need the following hypothesis.

(A) There is $\mu \in \mathbb{R}$ and a nonnegative progressively measurable process $\left\{f_{t}, t \geq 0\right\}$ such that $\langle\hat{y}, f(t, y)\rangle \leq f_{t}+\mu|y|$ for all $(t, y) \in \mathbb{R}_{+} \times \mathbb{R}^{N}$.

For a process $A \in \mathcal{V}$, we denote by $|A|_{t}$ its variation on the interval $[0, t]$.

LEMMA 3.1. Assume (A). Let $(Y, Z)$ be a solution of $B S D E(\xi, \sigma, f+\mathrm{d} A)$. If $Y \in \mathcal{D}^{p}$ and $E\left(\int_{0}^{\sigma} f_{t} \mathrm{~d} t\right)^{p}+E|A|_{\sigma}^{p}<\infty$ for some $p>0$, then $Z \in M^{p}$ and there exists $C_{p}$ depending only on $p$ such that for every $a \geq \mu$,

$$
\begin{aligned}
E\left(\int_{0}^{\sigma} \mathrm{e}^{2 a t}\left|Z_{t}\right|^{2} \mathrm{~d} t\right)^{p / 2} \leq & C_{p} E\left(\sup _{0 \leq t \leq \sigma} \mathrm{e}^{a p t}\left|Y_{t}\right|^{p}+\left(\int_{0}^{\sigma} \mathrm{e}^{a t} f_{t} \mathrm{~d} t\right)^{p}\right. \\
& \left.+\left(\int_{0}^{\sigma} \mathrm{e}^{a t} \mathrm{~d}|A|_{t}\right)^{p}\right)
\end{aligned}
$$

Proof. The proof goes through as for [3, Lemma 3.1], with obvious changes.

PROPOSITION 3.2. Assume (A). Let $(Y, Z)$ be a solution of $B \operatorname{SDE}(\xi, \sigma, f+\mathrm{d} A)$. If $Y \in \mathcal{D}^{p}$ and $E\left(\int_{0}^{\sigma} f_{t} \mathrm{~d} t\right)^{p}+E|A|_{\sigma}^{p}<\infty$ for some $p>1$, then there exists $C_{p}$ depending only on $p$, such that for every $a \geq \mu$,

$$
\begin{aligned}
& E\left(\sup _{0 \leq t \leq \sigma} \mathrm{e}^{a p t}\left|Y_{t}\right|^{p}+\left(\int_{0}^{\sigma} \mathrm{e}^{2 a t}\left|Z_{t}\right|^{2} \mathrm{~d} t\right)^{p / 2}\right) \\
& \leq C_{p} E\left(\mathrm{e}^{a p \sigma}|\xi|^{p}+\left(\int_{0}^{\sigma} \mathrm{e}^{a t} f_{t} \mathrm{~d} t\right)^{p}+\left(\int_{0}^{\sigma} \mathrm{e}^{a t} \mathrm{~d}|A|_{t}\right)^{p}\right) .
\end{aligned}
$$

Proof. It suffices to repeat, with obvious changes, arguments from the proof of [3, Proposition 3.2]. The only difference is that we use our Corollary 2.3 instead of [3, Corollary 2.3].

We now prove the analogues of [26, Theorem 2.2] and [4, Lemma 2.2] for equations with nonzero $\mathrm{d} A$.

LEMMA 3.3. Assume that

$$
\langle f(t, y), y\rangle \leq c|y|^{2}, \quad y \in \mathbb{R}^{N}, \quad t \geq 0
$$


for some $c \geq 0$ and $\|\xi\|_{\infty}+c+\left\||A|_{\sigma}\right\|_{\infty} \leq r<\infty$. If $(Y, Z)$ is a solution of $B \operatorname{SDE}(\xi, \sigma, f+\mathrm{d} A)$ such that $Y$ is of class $(D)$, then $\|Y\|_{\infty} \leq r$.

Proof. By Corollary 2.3,

$$
\left|Y_{t}\right| \leq|\xi|+\int_{t}^{\sigma}\left\langle f\left(s, Y_{s}\right), \hat{Y}_{s}\right\rangle \mathrm{d} s+\int_{t}^{\sigma}\left\langle\hat{Y}_{s}, \mathrm{~d} A_{s}\right\rangle-\int_{t}^{\sigma} Z_{s} \mathrm{~d} B_{s}, \quad t \geq 0 .
$$

Since $Y$ is of class (D), it follows from (3.1) that $\left|Y_{t}\right| \leq E^{\mathcal{F}_{t}}\left(|\xi|+c+|A|_{\sigma}\right), t \geq 0$, which implies the desired estimate.

LEMMA 3.4. Assume (A1)-(A3) are satisfied with $p=2$ and that $|f(t, y)| \leq$ $c+|f(t, 0)|$ for every $t \geq 0, y \in \mathbb{R}^{N}$. Then, there exists a unique solution $(Y, Z)$ of $\operatorname{BSDE}(\xi, \sigma, f+\mathrm{d} A)$ such that $(Y, Z) \in \mathcal{D}^{2} \otimes M^{2}$.

Proof. Using standard arguments, one can prove the existence of a unique solution $(\bar{Y}, \bar{Z}) \in \mathcal{D}^{2} \otimes M^{2}$ of the BSDE

$$
\bar{Y}_{t}=\int_{t}^{\sigma} \mathrm{d} A_{s}-\int_{t}^{\sigma} \bar{Z}_{s} \mathrm{~d} B_{s} .
$$

Furthermore, from [26], it follows that under the assumptions of the lemma, there exists a unique solution $\left(Y^{\prime}, Z^{\prime}\right) \in \mathcal{D}^{2} \otimes M^{2}$ of the BSDE

$$
Y_{t}^{\prime}=\xi+\int_{t}^{\sigma} \bar{f}\left(s, Y_{s}^{\prime}\right) \mathrm{d} s-\int_{t}^{\sigma} Z_{s}^{\prime} \mathrm{d} B_{s}, \quad t \in[0, \sigma]
$$

with $\bar{f}(t, y)=f\left(t, y+\bar{Y}_{t}\right)$. Set $(Y, Z)=\left(Y^{\prime}+\bar{Y}, Z^{\prime}+\bar{Z}\right)$. Then, $(Y, Z)$ is a solution of $\operatorname{BSDE}(\xi, \sigma, f+\mathrm{d} A)$.

We are now ready to formulate and prove the existence and uniqueness result in case $p>1$.

THEOREM 3.5. Let $p>1$. If (A1)-(A4) are satisfied, then there exists a unique solution $(Y, Z) \in \mathcal{D}^{p} \times M^{p}$ of $B S D E(\xi, \sigma, f+\mathrm{d} A)$.

Proof. Step 1. We first assume that $a \equiv\|f(\cdot, 0)\|_{\infty}+\|\xi\|_{\infty}+\left\||A|_{\sigma}\right\|_{\infty}<\infty$. Let us define $h_{n}$ as in the first step of the proof of [3, Theorem 4.2] (with $r>a$ ). Then, in much, the same way as in the proof of that theorem, but using Lemma 3.1 and Proposition 3.2 instead of [3, Lemma 3.1] and [3, Proposition 3.2] and Lemmas 3.3 and 3.4 instead of [26, Theorem 2.2] and [4, Lemma 2.2], one can prove that there exists a solution $(Y, Z) \in \mathcal{D}^{2} \otimes M^{2}$ of $\operatorname{BSDE}(\xi, \sigma, f+\mathrm{d} A)$.

Step 2. We define $\xi_{n}, f_{n}$ as in the second step of the proof of [3, Theorem 4.2] and set $A_{t}^{n}=\int_{0}^{t} \mathbf{1}_{\left\{|A|_{s} \leq n\right\}} \mathrm{d} A_{s}$. The proof of the existence of a solution $(Y, Z) \in \mathcal{D}^{p} \otimes M^{p}$ of $\operatorname{BSDE}(\xi, \sigma, f+\mathrm{d} A)$ goes as the proof of [3, Theorem 4.2], the only difference being in the use of Lemma 3.1 and Proposition 3.2 instead of [3, Lemma 3.2] and [3, Proposition 3.2].

We now turn to the case $p=1$. We first prove the uniqueness result. 
THEOREM 3.6. Let $p=1$. If(A2) is satisfied, then there exists at most one solution $(Y, Z)$ of $B \operatorname{SDE}(\xi, \sigma, f+\mathrm{d} A)$ such that $Y$ is of class $(D)$.

Proof Without the loss of generality, we may assume that $\mu \leq 0$. Let $(Y, Z)$, $\left(Y^{\prime}, Z^{\prime}\right)$ be the solutions of $\operatorname{BSDE}(\xi, \sigma, f+\mathrm{d} A)$ such that $Y, Y^{\prime}$ are of class (D). Then, $(\bar{Y}, \bar{Z})=\left(Y-Y^{\prime}, Z-Z^{\prime}\right)$ is a solution of the BSDE

$$
\bar{Y}_{t}=\int_{t}^{\sigma} f\left(s, Y_{s}\right)-f\left(s, Y_{s}^{\prime}\right) \mathrm{d} s-\int_{t}^{\sigma} \bar{Z}_{s} h B_{s}, \quad t \geq 0 .
$$

Let $\tau_{k}=\inf \left\{t \geq 0 ; \int_{0}^{t}\left|\bar{Z}_{s}\right|^{2} \mathrm{~d} s \geq k\right\}$. By the Itô-Meyer formula and (A2),

$$
\begin{aligned}
\left|\bar{Y}_{t}\right| \leq & \left|\bar{Y}_{\tau_{k} \wedge \sigma}\right|+\int_{t}^{\tau_{k} \wedge \sigma}\left\langle f\left(s, Y_{s}\right)-f\left(s, Y_{s}^{\prime}\right), \operatorname{sg} n\left(\bar{Y}_{s}\right)\right\rangle \mathrm{d} s \\
& -\int_{t}^{\tau_{k} \wedge \sigma}\left\langle\operatorname{sgn}\left(\bar{Y}_{s}\right), \bar{Z}_{s} \mathrm{~d} B_{s}\right\rangle \leq-\int_{t}^{\tau_{k} \wedge \sigma}\left\langle\operatorname{sg} n\left(\bar{Y}_{s}\right), \bar{Z}_{s} \mathrm{~d} B_{s}\right\rangle, \quad t \geq 0 .
\end{aligned}
$$

Taking the conditional expectation with respect to $\mathcal{F}_{t}$ on both sides of the above inequality and then letting $k \rightarrow \infty$ and using the fact that $\bar{Y}$ is of class (D), we conclude that $\left|\bar{Y}_{t}\right|=0, t \geq 0$.

For $k>0$, let us put

$$
T_{k}(y)=\frac{k y}{k \vee|y|}, \quad y \in \mathbb{R}^{N} .
$$

THEOREM 3.7. Let $p=1$. If (A1)-(A4) are satisfied, then there exists a solution $(Y, Z)$ of $B S D E(\xi, \sigma, f+\mathrm{d} A)$ such that $(Y, Z) \in \mathcal{D}^{q} \otimes M^{q}$ for $q \in(0,1)$ and $Y$ is of class (D).

Proof. Without the loss of generality, we may assume that $\mu \leq 0$. Set

$\xi^{n}=T_{n}(\xi), \quad f_{n}(t, y)=f(t, y)-f(t, 0)+T_{n}(f(t, 0)), \quad A_{t}^{n}=\int_{0}^{t} \mathbf{1}_{\left\{|A|_{s} \leq n\right\}} \mathrm{d} A_{s}$.

By Theorem 3.5, for every $n \in \mathbb{N}$, there exists a solution $\left(Y^{n}, Z^{n}\right) \in \mathcal{D}^{2} \otimes M^{2}$ of $\operatorname{BSDE}\left(\xi^{n}, \sigma, f_{n}+\mathrm{d} A^{n}\right)$. Let $m \geq n$. Write $\delta Y=Y^{m}-Y^{n}, \delta Z=Z^{m}-Z^{n}, \delta \xi=$ $\xi^{m}-\xi^{n}$ and

$$
\tau_{k}=\inf \left\{t \geq 0 ; \int_{0}^{t}\left|\delta Z_{s}\right|^{2} \mathrm{~d} s>k\right\} .
$$

By the Itô-Meyer formula, for $t \geq 0$, we have

$$
\begin{aligned}
\left|\delta Y_{t \wedge \tau_{k}}\right| \leq & \left|\delta Y_{\tau_{k} \wedge \sigma}\right|+\int_{t}^{\tau_{k} \wedge \sigma}\left\langle\operatorname{sgn}\left(\delta Y_{s}\right), f_{m}\left(s, Y_{s}^{m}\right)-f_{n}\left(s, Y_{s}^{n}\right)\right\rangle \mathrm{d} s \\
& +\int_{t}^{\tau_{k} \wedge \sigma}\left\langle\operatorname{sg} n\left(\delta Y_{s-}\right), d\left(A_{s}^{m}-A_{s}^{n}\right)\right\rangle+\int_{t}^{\tau_{k} \wedge \sigma}\left\langle\operatorname{sg} n\left(\delta Y_{s}\right), \delta Z_{s} \mathrm{~d} B_{s}\right\rangle \\
\leq & \left|\delta Y_{\tau_{k} \wedge \sigma}\right|+\int_{t}^{\tau_{k} \wedge \sigma}\left|f_{m}\left(s, Y_{s}^{n}\right)-f_{n}\left(s, Y_{s}^{n}\right)\right| \mathrm{d} s \\
& +\int_{t}^{\tau_{k} \wedge \sigma} \mathrm{d}\left|A^{m}-A^{n}\right|_{s}+\int_{t}^{\tau_{k} \wedge \sigma}\left\langle\operatorname{sg} n\left(\delta Y_{s}\right), \delta Z_{s} \mathrm{~d} B_{s}\right\rangle,
\end{aligned}
$$


the last inequality being a consequence of monotonicity of $f_{n}$ with respect to $y$. Conditioning with respect to $\mathcal{F}_{t}$ and using the fact that $\delta Y$ is of class (D), we conclude from the above inequality that

$$
\left|\delta Y_{t}\right| \leq E^{\mathcal{F}_{t}}\left(|\xi| \mathbf{1}_{\{|\xi|>n\}}+\int_{0}^{\sigma}|f(s, 0)| \mathbf{1}_{\{|f(s, 0)|>n\}} \mathrm{d} s+\int_{0}^{\sigma} \mathbf{1}_{\left\{|A|_{s}>n\right\}} \mathrm{d}|A|_{s}\right)
$$

for $t \geq 0$. To complete the proof, it suffices now to repeat step by step the arguments following Eq. (12) in the proof of [3, Proposition 6.4].

\section{Markov processes and potential theory}

To make our exposition in the next sections self-contained, in this section, we recall some useful facts about diffusions associated with the operator $A_{t}$ defined by (1.2), their additive functionals and the Revuz correspondence between these functionals and soft measures.

\subsection{Time-inhomogeneous diffusions}

Let $\Omega=C\left(\mathbb{R}_{+} ; \mathbb{R}^{d}\right)$ be the space of continuous $\mathbb{R}^{d}$-valued functions on $\mathbb{R}_{+}=$ $[0, \infty), X$ be the canonical process on $\Omega, \mathcal{F}_{s, t}^{0}=\sigma\left(X_{u}, u \in[s, t]\right)$. We define $\mathcal{F}_{s, \infty}$ as the completion of $\mathcal{F}_{s, \infty}^{0}$ with respect to the family $\mathcal{P}=\left\{P_{s, \mu}: \mu\right.$ is a probability measure on $\left.\mathcal{B}\left(\mathbb{R}^{d}\right)\right\}$, where $P_{s, \mu}(\cdot)=\int_{\mathbb{R}^{d}} P_{s, x}(\cdot) \mu(\mathrm{d} x)$, and then we define $\mathcal{F}_{s, t}$ as the completion of $\mathcal{F}_{s, t}^{0}$ in $\mathcal{F}_{s, \infty}$ with respect to $\mathcal{P}$.

Let $p$ denote the fundamental solution for the operator $A_{t}$ defined by (1.2). It is known (see [31]) that there exists a unique time-inhomogeneous Markov process $\mathbb{X}=\left\{\left(X, P_{s, x}\right):(s, x) \in \mathbb{R}_{+} \times \mathbb{R}^{d}\right\}$ associated with $A_{t}$. Namely, $\mathbb{X}$ is a unique Markov process for which $p$ is the transition density function, i.e.,

$$
P_{s, x}\left(X_{t}=x ; 0 \leq t \leq s\right)=1, \quad P_{s, x}\left(X_{t} \in \Gamma\right)=\int_{\Gamma} p(s, x, t, y) d y, \quad t>s
$$

for any $\Gamma \in \mathcal{B}\left(\mathbb{R}^{d}\right)$. It is known (see [32]) that $\mathbb{X}$ admits the so-called strict Fukushima decomposition, i.e., for every $(s, x) \in \mathbb{R}_{+} \times \mathbb{R}^{d}$,

$$
X_{t}-X_{s}=A_{s, t}+M_{s, t}, \quad s \leq t, \quad P_{s, x} \text {-a.s. }
$$

where $M$ is a two-parameter martingale additive functional (MAF) of $\mathbb{X}$ of finite energy and $A$ is a two-parameter continuous additive functional (CAF) of $\mathbb{X}$ of zero energy. Moreover,

$$
\left\langle M_{s, \cdot}^{i}, M_{s, \cdot}^{j}\right\rangle_{t}=\int_{s}^{t} a_{i j}\left(r, X_{r}\right) \mathrm{d} r, \quad s \leq t,
$$

which implies in particular that the process

$$
B_{s, t}=\int_{s}^{t} \sigma^{-1}\left(r, X_{r}\right) \mathrm{d} M_{s, r}, \quad t \geq s
$$


where $\sigma \cdot \sigma^{T}=a$ is a Brownian motion under $P_{S, x}$. It is also known (see [20]) that $B_{s, \text { is }}$ an $\left\{\mathcal{F}_{s, t}\right\}_{t \geq s}$-Brownian motion.

\subsection{Time-homogeneous diffusions}

In what follows we will also make substantial use of time-homogeneous Markov process $\mathbb{X}^{\prime}$ associated with the operator $\frac{\partial}{\partial t}+A_{t}$ (see [24]). A brief sketch of a useful construction of $\mathbb{X}^{\prime}$ on an extension of $\Omega$ is given below.

We set

$$
\Omega^{\prime}=\mathbb{R}_{+} \times \Omega, \quad P_{s, x}^{\prime}(B)=P_{s, x}(\{\omega \in \Omega:(s, \omega) \in B\})
$$

and consider the process $\mathbf{X}$ on $\Omega^{\prime}$ defined as

$$
\mathbf{X}_{t}(s, \omega)=\left(s+t, X_{s+t}(\omega)\right), \quad t \geq 0
$$

Let $\mathcal{F}_{t}^{\prime 0}=\sigma\left(\mathbf{X}_{u}, u \leq t\right), \mathcal{F}_{\infty}^{\prime 0}=\sigma\left(\mathbf{X}_{u}, u<\infty\right)$ and let $\mathcal{F}_{\infty}^{\prime}$ denote the completion of $\mathcal{F}_{\infty}^{\prime 0}$ with respect to the family $\mathcal{P}^{\prime}=\left\{P_{\mu}^{\prime}: \mu\right.$ is a probability measure on $\mathbb{R}_{+} \times$ $\left.\mathbb{R}^{d}\right\}$ and $\mathcal{F}_{t}^{\prime}$ denote the completion of $\mathcal{F}_{t}^{\prime 0}$ in $\mathcal{F}_{\infty}^{\prime}$ with respect to $\mathcal{P}^{\prime}$. Then, $\mathbb{X}^{\prime}=$ $\left\{\left(\mathbf{X}_{t}, P_{s, x}^{\prime}\right) ;(s, x) \in \mathbb{R}_{+} \times \mathbb{R}^{d}\right\}$ is a time-homogeneous Markov process with respect to the filtration $\left\{\mathcal{F}_{t}^{\prime}\right\}$ with the transition density

$$
P^{\prime}(t,(s, x), \Gamma)=P\left(s, x, s+t, \Gamma_{s+t}\right),
$$

where $\Gamma_{s+t}=\left\{x \in \mathbb{R}^{d}:(s+t, x) \in \Gamma\right\}$.

It is known (see [25]) that $\mathbb{X}^{\prime}$ admits the strict Fukushima decomposition, i.e., for every $(s, x) \in \mathbb{R}_{+} \times \mathbb{R}^{d}$,

$$
\mathbf{X}_{t}=\mathbf{X}_{0}+\mathbf{A}_{t}+\mathbf{M}_{t}, \quad t \geq 0, \quad P_{s, x}^{\prime} \text {-a.s. }
$$

where $\mathbf{A}$ is a CAF of $\mathbb{X}^{\prime}$ of zero energy and $\mathbf{M}$ is a MAF of $\mathbb{X}^{\prime}$ of finite energy. It is also known (see [19]) that

$$
p\left(\mathbf{A}_{t}\right)\left(\omega^{\prime}\right)=A_{s, s+t}(\omega), \quad p\left(\mathbf{M}_{t}\right)\left(\omega^{\prime}\right)=M_{s, s+t}(\omega), \quad t \geq 0
$$

for $\omega^{\prime}=(s, \omega)$, where $p: \mathbb{R}_{+} \times \mathbb{R}^{d} \rightarrow \mathbb{R}^{d}$ is the orthogonal projection. Set

$$
A_{t}=p\left(\mathbf{A}_{t}\right), \quad M_{t}=p\left(\mathbf{M}_{t}\right), \quad t \geq 0
$$

and for $t \geq 0$ define $\tau(t): \Omega^{\prime} \rightarrow \mathbb{R}_{+}$by putting

$$
\tau(t)\left(\omega^{\prime}\right)=s+t=\tau(0)(\omega)+t
$$

for $\omega^{\prime}=(s, \omega)$. From now on, we adopt the convention that if $\xi$ is a random variable on $\Omega$, then $\xi\left(\omega^{\prime}\right)=\xi(\omega)$ for $\omega^{\prime}=(s, \omega) \in \Omega^{\prime}$. With this convention

$$
\mathbf{X}_{t}=\left(\tau(t), X_{\tau(t)}\right), \quad t \geq 0
$$


We put

$$
\zeta=\inf \left\{t \geq 0 ; \mathbf{X}_{t} \notin \mathbb{R}_{+} \times D\right\}, \quad \zeta_{\tau}=\zeta \wedge T_{\tau}, \quad T_{\tau}=T-\tau(0)
$$

Set

$$
B_{t}=\int_{0}^{t} \sigma^{-1}\left(\mathbf{X}_{r}\right) \mathrm{d} M_{r}, \quad t \geq 0 .
$$

By (4.1) and (4.6),

$$
\left\langle M^{i}, M^{j}\right\rangle_{t}=\int_{0}^{t} a_{i j}\left(\mathbf{X}_{r}\right) \mathrm{d} r, \quad t \geq 0, \quad P_{s, x}^{\prime} \text {-a.s. }
$$

for every $(s, x) \in \mathbb{R}_{+} \times \mathbb{R}^{d}$. Therefore, $B$ is a Brownian motion under $P_{s, x}^{\prime}$ for every $(s, x) \in \mathbb{R}_{+} \times \mathbb{R}^{d}$. In fact, by [20], it is an $\left\{\mathcal{F}_{t}^{\prime}\right\}_{t \geq 0}$-Brownian motion.

4.3. Capacity, soft measures, and quasi-continuity

Let $\mathcal{W}$ be the space of all $u \in L^{2}\left(\mathbb{R}_{+} ; H_{0}^{1}(D)\right)$ such that $\frac{\partial u}{\partial t} \in L^{2}\left(\mathbb{R}_{+} ; H^{-1}(D)\right)$ endowed with the usual norm $\|u\|_{\mathcal{W}}=\|u\|_{L^{2}\left(\mathbb{R}_{+} ; H_{0}^{1}(D)\right)}+\left\|\frac{\partial u}{\partial t}\right\|_{L^{2}\left(\mathbb{R}_{+} ; H^{-1}(D)\right)}$.

We define the parabolic capacity of an open set $U \subset \mathbb{R}_{+} \times D$ as

$$
\operatorname{cap}(U)=\inf \left\{\|u\|_{\mathcal{W}}: u \in \mathcal{W}, u \geq \mathbf{1}_{U} \text { a.e. in } \mathbb{R}_{+} \times D\right\} .
$$

The parabolic capacity of a Borel set $B \subset \mathbb{R}_{+} \times D$ is defined as

$$
\operatorname{cap}(B)=\inf \left\{\operatorname{cap}(U): U \text { is an open subset of } \mathbb{R}_{+} \times D, B \subset U\right\} .
$$

We also consider the Newtonian capacity on $D$. For an open set $U \subset D$, we put

$$
\operatorname{cap}_{N}(U)=\inf \left\{\|u\|_{H_{0}^{1}(D)}: u \in H_{0}^{1}(D), u \geq \mathbf{1}_{U} \text { a.e. in } D\right\},
$$

and for a Borel set $B \subset D$, we put

$$
\operatorname{cap}_{N}(B)=\inf \left\{\operatorname{cap}_{N}(U): U \text { is an open subset of } D, B \subset U\right\} \text {. }
$$

From now on, we say that some property is satisfied for quasi-every (q.e. for short) $x \in D$ (respectively, $\left.(s, x) \in \mathbb{R}_{+} \times D\right)$ if it is satisfied except for some Borel subset of $D$ (respectively, $\mathbb{R}_{+} \times D$ ) of cap $_{N}$ (respectively, cap) capacity zero.

Let $\mu$ be a Radon measure on $D$ (respectively, $\mathbb{R}_{+} \times D$ ). Following [6], we say that $\mu$ is soft if $\mu$ charges no set of cap ${ }_{N}$ (respectively, cap) capacity zero. In what follows by $\mathcal{M}_{0}(D)$ (respectively, $\mathcal{M}_{0}$ ), we denote the set of all soft measures on $D$ (respectively, $\mathbb{R}_{+} \times D$ ) and by $\mathcal{M}_{0, b}(D)$ (respectively, $\mathcal{M}_{0, b}$ ) the set of all bounded soft measures on $D$ (respectively, $\mathbb{R}_{+} \times D$ ).

Let $B$ be a Borel subset of $\mathbb{R}_{+} \times D$ (respectively, $D$ ) and $u: B \rightarrow \mathbb{R}$ be a Borel measurable function. We say that $u$ is quasi-continuous if for every $\varepsilon>0$, there exists a closed set $F_{\varepsilon} \subset B$ such that $\operatorname{cap}\left(B \backslash F_{\varepsilon}\right)<\varepsilon$ (respectively, $\operatorname{cap}_{N}\left(B \backslash F_{\varepsilon}\right)<\varepsilon$ ) such that $u_{\mid F_{\varepsilon}}$ is continuous. In the paper, we shall mostly work with functions on $B=D$ 
or $B=D_{T}$. We adopt the convention that for $u$ defined on $B=D$ ( respectively, $\left.B=D_{T}\right) u(x)=0$ (respectively, $u(t, x)=0$ ) for $x \in \mathbb{R}^{d} \backslash D$ (respectively, $\left.(t, x) \in\left(\mathbb{R}_{+} \times \mathbb{R}^{d}\right) \backslash D_{T}\right)$. It is well known that $u$ on $D_{T}$ (respectively, $\left.D\right)$ is quasicontinuous iff the process $\left[0, \zeta_{\tau}\right) \ni t \rightarrow u\left(\mathbf{X}_{t}\right)$ (respectively, $\left[0, \zeta^{0}\right) \ni t \rightarrow u\left(X_{t}\right)$ ) is continuous under $P_{s, x}^{\prime}$ (respectively, $P_{0, x}$ ) for q.e. $(s, x) \in D_{T}$ (respectively, q.e. $x \in D)$. We will also consider quasi-càdlàg functions on $D_{T}$, i.e., Borel functions $u$ on $D_{T}$, such that the process $\left[0, \zeta_{\tau}\right] \ni t \rightarrow u\left(\mathbf{X}_{t}\right)$ is càdlàg for q.e. $(s, x) \in D_{T}$.

\subsection{Additive functionals and soft measures}

Let $E_{s, x}$ (respectively, $E_{s, x}^{\prime}$ ) denote the expectation with respect to $P_{s, x}$ (respectively, $P_{s, x}^{\prime}$ ) and let $m_{1}$ denote the Lebesgue measure on $\mathbb{R}_{+} \times \mathbb{R}^{d}$. Let $\zeta^{0}$ be defined by (1.7) with $s=0$, i.e.,

$$
\zeta^{0}=\inf \left\{t \geq 0, X_{t} \notin D\right\}
$$

Let us recall that a positive $\mathrm{AF} A$ of $\mathbb{X}^{\prime}$ and a positive soft measure $\mu$ on $\mathbb{R}_{+} \times D$ are in the Revuz correspondence if

$$
\langle\mu, f\rangle \equiv \int_{0}^{\infty} \int_{D} f \mathrm{~d} \mu=\lim _{\alpha \rightarrow \infty} \alpha \int_{0}^{\infty} \int_{D}\left(E_{s, x}^{\prime} \int_{0}^{\zeta} \mathrm{e}^{-\alpha t} f\left(\mathbf{X}_{t}\right) \mathrm{d} A_{t}\right) \mathrm{d} m_{1}(s, x)
$$

for every $f \in \mathcal{B}^{+}\left(\mathbb{R}_{+} \times D\right)$. If $\langle\mu, 1\rangle<\infty$, then $A$ is called integrable. It is known (see $[22,30])$ that under $(4.8)$, the family of all integrable positive AFs of $\mathbb{X}^{\prime}$ and the family of all bounded positive soft measures on $\mathbb{R}_{+} \times D$ are in one-to-one correspondence. In what follows the additive functional corresponding to a positive bounded soft measure $\mu$ will be denoted by $A^{\mu}$.

Let $p_{D}^{\prime}$ denote the transition density of the process $\mathbb{X}^{\prime}$ killed on exiting $\mathbb{R}_{+} \times D$. It is known that $A^{\mu}$ corresponds to $\mu$ iff for q.e. $(s, x) \in \mathbb{R}_{+} \times D$,

$$
E_{s, x}^{\prime} \int_{0}^{\zeta} f\left(\mathbf{X}_{t}\right) \mathrm{d} A_{t}^{\mu}=\int_{0}^{\infty}\left(\int_{0}^{\infty} \int_{D} f(z) p_{D}^{\prime}(t,(s, x), z) \mathrm{d} \mu(z)\right) \mathrm{d} t
$$

for every $f \in \mathcal{B}_{+}\left(\mathbb{R}^{+} \times D\right)$ (see [22]).

Suppose now that the coefficients of the operator (1.2) do not depend on time, i.e., $a_{i j}(t, x)=a_{i j}(x)$ for $(t, x) \in D_{T}$. We then set

$$
A=\sum_{i j=1}^{d} \frac{\partial}{\partial x_{j}}\left(a_{i j} \frac{\partial}{\partial x_{i}}\right)
$$

It is well known that the fundamental solution for $A$ has the property that $p(s, x, t, y)=$ $p(t-s, x, y)$ for any $t>s, x \neq y$. Consequently, $\mathbb{X}=\left\{\left(X, P_{x}\right): x \in \mathbb{R}^{d}\right\}$, where $P_{x}=P_{0, x}^{\prime}$ is a time-homogeneous Markov process with the transition density $p(t, x, y)=p(0, x, t, y)$. It is also known (see, e.g., [9]) that in the time-homogeneous 
case, a positive CAF $A$ of $\mathbb{X}$ and a positive soft measure $\mu$ on $D$ are in the Revuz correspondence if

$$
\langle\mu, f\rangle=\lim _{\alpha \rightarrow \infty} \alpha \int_{D}\left(E_{x} \int_{0}^{\zeta^{0}} \mathrm{e}^{-\alpha t} f\left(X_{t}\right) \mathrm{d} A_{t}\right) \mathrm{d} m(x)
$$

for every $f \in \mathcal{B}^{+}(D)$, where $E_{x}$ denotes the expectation with respect to $P_{x}$. If $\langle\mu, 1\rangle<$ $\infty$, then $A$ is called integrable. In [9], it is proved that the family of all integrable positive CAFs of $\mathbb{X}$ and the family of all bounded positive soft measures on $D$ are in one-to-one correspondence via formula (4.11).

Let $A^{\mu}$ denote the additive functional corresponding to a positive bounded soft measure $\mu, p_{D}$ denote the transition density of the process $\mathbb{X}$ killed on exiting $D$, and let $G_{D}(x, y)=\int_{0}^{\infty} p_{D}(t, x, y) \mathrm{d} t$ be the Green function for $D$. Then, for q.e. $x \in D$,

$E_{x} \int_{0}^{\zeta^{0}} f\left(X_{t}\right) \mathrm{d} A_{t}^{\mu}=\int_{0}^{\infty} \int_{D} f(y) p_{D}(t, x, y) \mathrm{d} \mu(y) \mathrm{d} t=\int_{D} f(y) G_{D}(x, y) \mathrm{d} \mu(y)$

for every $f \in \mathcal{B}^{+}(D)$. In the whole paper for a fixed Borel positive measure $\mu$ on $D_{T}$ (respectively, $D$ ), we denote

$R^{0, T} \mu(s, x)=\int_{0}^{\infty}\left(\int_{0}^{T} \int_{D} p_{D}^{\prime}(t,(s, x), z) \mathrm{d} \mu(z)\right) \mathrm{d} t, \quad R \mu(x)=\int_{D} G_{D}(x, y) \mathrm{d} \mu(y)$.

Finally, let us recall that there is $c$ depending only on $d, \Lambda$ such that $p(t, x, y) \leq$ $C t^{-d / 2}$ for $t>0, x, y \in \mathbb{R}^{d}$ (see, e.g., [1]). Therefore, by [5, Theorem 1.17], there is $c$ depending only on $d, \Lambda$ such that

$$
\sup _{x \in \mathbb{R}^{d}} E_{x} \zeta^{0} \leq c|D|^{d / 2}
$$

where $|D|$ denotes the Lebesgue measure of $D$, whereas from Corollary to Proposition 1.18 in [5], it follows that there exists constants $a>0, b>0$ depending only on $d, \Lambda$ and $|D|$ such that for every $t>0$,

$$
\sup _{x \in \mathbb{R}^{d}} P_{x}\left(\zeta^{0}>t\right) \leq a \mathrm{e}^{-b t}
$$

\section{Markov-type BSDEs and PDEs}

Let us fix $T>0$ and set $D_{T}=[0, T] \times D$. In this section, we show existence and uniqueness results for systems of PDEs of the form

$$
\left\{\begin{array}{l}
\frac{\partial u^{k}}{\partial t}+A_{t} u^{k}=-f^{k}(t, x, u)-\mu^{k}, \quad k=1, \ldots, N \\
u_{\mid \partial D}(t, \cdot)=0, t \in[0, T), \quad u(T, \cdot)=\varphi \text { on } D
\end{array}\right.
$$

where $A_{t}$ is given by (1.2).

Let $f: D_{T} \times \mathbb{R}^{N} \rightarrow \mathbb{R}^{N}$. We consider the following hypotheses. 
(H1) $f(\cdot, \cdot, y)$ is measurable for every $y \in \mathbb{R}^{N}$ and $f(t, x, \cdot)$ is continuous for a.e. $(t, x) \in D_{T}$.

(H2) There is $\alpha \in \mathbb{R}$ such that $\left\langle f(t, x, y)-f\left(t, x, y^{\prime}\right), y-y^{\prime}\right\rangle \leq \alpha\left|y-y^{\prime}\right|^{2}$ for every $y, y^{\prime} \in \mathbb{R}^{N}$ and a.e. $(t, x) \in D_{T}$.

(H3) $f(\cdot, \cdot, 0) \in L^{1}\left(D_{T}\right), \mu \in \mathcal{M}_{0, b}\left(D_{T}\right), \varphi \in L^{1}(D)$.

(H4) $\forall_{r>0, y \in \mathbb{R}^{N}} R^{0, T}\left(\sup _{|y| \leq r}|f(\cdot, \cdot, y)|\right)<\infty, m_{1}$-a.e.

REMARK 5.1. It is known (see [17, Proposition 3.6]) that if $f \in L^{1}\left(D_{T}\right)$, then $R^{0, T}|f|<\infty, m_{1}$-a.e. Therefore, (H4) is satisfied if $\sup _{|y| \leq r}|f(\cdot, \cdot, y)| \in L^{1}\left(D_{T}\right)$ for every $y \in \mathbb{R}^{N}$ and $r \geq 0$. However, the class of functions $f \in \mathcal{B}\left(D_{T}\right)$ such that $R^{0, T}|f|<\infty, m_{1}$-a.e. is wider than $L^{1}\left(D_{T}\right)$. It includes in particular the space $L^{1}\left(D_{T} ; \delta \cdot m_{1}\right)$, where $\delta(x)=\operatorname{dist}(x, \partial D)$ (see [17, Example 5.2]).

It is known that in case $N=1$, one can find solutions of problems of the form (5.1) in the (nonlinear) space $\mathcal{T}_{2}^{0,1}$ of all Borel measurable functions $u$ on $D_{T}$ such that $T_{k}(u) \in L^{2}\left(0, T ; H_{0}^{1}(D)\right)$ for every $k \geq 1$ (see [2,6]). In the case of systems, the problem is more difficult, because we do not know whether the solutions or its truncation have gradients in the usual sense (i.e., locally in some Sobolev space). This is related to the lack of integrability of $f_{u}$. The same problem appears in the case of elliptic systems. In the scalar case, it is known that a solution of (5.1) belongs to $W_{0}^{1, q}(D)$ for every $q \in\left[1, \frac{d}{d-1}\right.$ ) (see [34]) but in case $N>1$, the problem whether a solution belongs to $W_{0}^{1, q}(D)$ for some $q \geq 1$ is open. To overcome the difficulty in [15], the existence and uniqueness of elliptic systems with measure data similar to (5.1) is proved in some wider than $\mathcal{T}_{2}^{0,1}$ (see Corollary 5.6) linear space. The space introduced in [15] makes essential use of the Markov process $\mathbb{X}$ associated with the operator $A$ defined by (4.10) and therefore may be called a stochastic Sobolev space. In what follows we extend the ideas from [15] to parabolic systems. We begin with the definition of the stochastic Sobolev space of functions depending on time.

Let $W^{0,1}\left(\mathbb{X}^{D_{T}}\right)$ denote the set of all $u \in F M$ (definition below) for which there exists a sequence $\left\{u_{n}\right\} \subset C_{c}^{\infty}\left(D_{T}\right)$ such that for q.e. $(s, x) \in D_{T}$,

$$
\int_{0}^{\zeta_{\tau}}\left|\left(u_{n}-u\right)\left(\mathbf{X}_{t}\right)\right|^{2} \mathrm{~d} t \rightarrow 0 \quad \text { in probability } P_{s, x}^{\prime} \text { as } n \rightarrow \infty
$$

and

$$
\int_{0}^{\zeta_{\tau}}\left|\nabla\left(u_{n}-u_{m}\right)\left(\mathbf{X}_{t}\right)\right|^{2} \mathrm{~d} t \rightarrow 0 \quad \text { in probability } P_{s, x}^{\prime} \text { as } n, m \rightarrow \infty .
$$

In [14], it is proved that for every $u \in W^{0,1}\left(\mathbb{X}^{D_{T}}\right)$, there exists a unique (a.e.) function $v \in \mathcal{B}\left(D_{T}\right)$ such that for every $\left\{u_{n}\right\} \subset C_{c}^{\infty}\left(D_{T}\right)$ satisfying (5.2) and (5.3),

$$
\int_{0}^{\zeta_{\tau}}\left|\nabla u_{n}\left(\mathbf{X}_{t}\right)-v\left(\mathbf{X}_{t}\right)\right|^{2} \mathrm{~d} t \rightarrow 0 \quad \text { in probability } P_{s, x}^{\prime} \text { as } n \rightarrow \infty
$$

for q.e. $(s, x) \in D_{T}$. 
Given $u \in W^{0,1}\left(\mathbb{X}^{D_{T}}\right)$, we denote by $\nabla_{\mathbb{X}} u$ the unique function $v$ satisfying (5.4). Notice that directly from the construction of $\nabla_{\mathbb{X}} u$, it follows that $\nabla_{\mathbb{X}} u=\nabla u$ a.e. if $u \in L^{2}\left(0, T ; H_{0}^{1}(D)\right)$.

By $F M$, we denote the space of Borel measurable functions $u$ on $D_{T}$ such that for q.e. $(s, x) \in D_{T}, P_{s, x}^{\prime}\left(\int_{0}^{\zeta_{\tau}}\left|u\left(\mathbf{X}_{r}\right)\right|^{2} \mathrm{~d} r<\infty\right)=1$. We say that $u_{n} \rightarrow u$ in $F M$ if (5.2) holds for q.e. $(s, x) \in D_{T}$.

DEFINITION. Let $u_{n}, u \in W^{0,1}\left(\mathbb{X}^{D_{T}}\right)$. We say that $u_{n} \rightarrow u$ in $W^{0,1}\left(\mathbb{X}^{D_{T}}\right)$ if $u_{n} \rightarrow u$ in $F M$ and $\nabla_{\mathbb{X}} u_{n} \rightarrow \nabla_{\mathbb{X}} u$ in $F M$.

REMARK 5.2. (i) Let $\mathcal{O}(\rightarrow)$ be the topology generated by the convergence in $W^{0,1}\left(\mathbb{X}^{D_{T}}\right)$. This topology is metrizable by the F-norm

$$
|u|_{1}=E_{m_{1}}\left[\left(\int_{0}^{\zeta_{\tau}} u^{2}\left(\mathbf{X}_{r}\right) \mathrm{d} r\right)^{1 / 2} \wedge 1\right]+E_{m_{1}}\left[\left(\int_{0}^{\zeta_{\tau}}\left|\nabla_{\mathbb{X}} u\right|^{2}\left(\mathbf{X}_{r}\right) \mathrm{d} r\right)^{1 / 2} \wedge 1\right] .
$$

Indeed, by the Kantorovich-Kisyński theorem (see [11,12]),

$$
u_{n} \rightarrow u \text { in } \mathcal{O}(\rightarrow) \text { iff } \forall\left(n_{k}\right) \subset(n) \exists\left(n_{k_{l}}\right) \subset\left(n_{k}\right) \quad u_{n_{k_{l}}} \rightarrow u \text { in } W^{0,1}\left(\mathbb{X}^{D_{T}}\right)
$$

Assume that $u_{n} \rightarrow u$ in $\mathcal{O}(\rightarrow)$ and $\left(u_{n}\right)$ does not converge to $u$ in $|\cdot|_{1}$. Then, there exists $\varepsilon>0$ and subsequence $\left(n_{k}\right) \subset(n)$ such that

$$
\left|u-u_{n_{k}}\right|_{1}>\varepsilon, \quad k \geq 1 .
$$

On the other hand, by (5.5), there exists a subsequence $\left(n_{k_{l}}\right) \subset\left(n_{k}\right)$ such that $u_{n_{k_{l}}} \rightarrow u$ in $W^{0,1}\left(\mathbb{X}^{D_{T}}\right)$. Hence, by the Lebesgue dominated convergence theorem, $\left|u_{n_{k_{l}}}-u\right|_{1} \rightarrow 0$ as $l \rightarrow \infty$, which contradicts (5.6). Now, assume that $\left|u_{n}-u\right|_{1} \rightarrow 0$ as $n \rightarrow \infty$ and let $\left(n_{k}\right) \subset(n)$. By [16, Proposition 3.3], there exists a subsequence $\left(n_{k_{l}}\right) \subset\left(n_{k}\right)$ such that $u_{n_{k_{l}}} \rightarrow u$ in $W^{0,1}\left(\mathbb{X}^{D_{T}}\right)$. Since $\left(n_{k}\right) \subset(n)$ was arbitrary, (5.5) implies that $u_{n} \rightarrow u$ in $\mathcal{O}(\rightarrow)$.

(ii) By $\left[14\right.$, Proposition 4.6], the space $\left(W^{0,1}\left(\mathbb{X}^{D_{T}}\right), \mathcal{O}(\rightarrow)\right)$ is complete.

LEMMA 5.3. If $u \in W^{0,1}\left(\mathbb{X}^{D_{T}}\right), \theta \in C^{1}(\mathbb{R})$ and there is $c>0$ such that $\left|\theta^{\prime}(t)\right| \leq$ $c$ for $t \in \mathbb{R}$, then $\theta(u) \in W^{0,1}\left(\mathbb{X}^{D_{T}}\right)$ and $\nabla_{\mathbb{X}}(\theta(u))=\theta^{\prime}(u) \nabla_{\mathbb{X}} u$.

Proof. Since $u \in W^{0,1}\left(\mathbb{X}^{D_{T}}\right)$, there exists a sequence $\left\{\eta_{n}\right\} \subset C_{c}\left(D_{T}\right)$ such that $\eta_{n} \rightarrow u$ and $\nabla \eta_{n} \rightarrow \nabla_{\mathbb{X}} u$ in $F M$. Using the assumptions on $\theta$, one can easily show that $\theta\left(\eta_{n}\right) \rightarrow \theta(u)$ and $\nabla \theta\left(\eta_{n}\right)=\theta^{\prime}\left(\eta_{n}\right) \nabla \eta_{n} \rightarrow \theta^{\prime}(u) \nabla_{\mathbb{X}} u$ in $F M$, which proves the desired result.

LEMMA 5.4. Let $k \in \mathbb{R}$ and $u \in W^{0,1}\left(\mathbb{X}^{D_{T}}\right)$. Then, $u \wedge k, u \vee k \in W^{0,1}\left(\mathbb{X}^{D_{T}}\right)$ and

$$
\begin{aligned}
& \nabla_{\mathbb{X}}(u \wedge k)=\mathbf{1}_{(-\infty, k)}(u) \nabla_{\mathbb{X}} u=\mathbf{1}_{(-\infty, k]}(u) \nabla_{\mathbb{X}} u, \text { a.e., } \\
& \nabla_{\mathbb{X}}(u \vee k)=\mathbf{1}_{(k, \infty)}(u) \nabla_{\mathbb{X}} u=\mathbf{1}_{[k, \infty)}(u) \nabla_{\mathbb{X}} u, \quad \text { a.e. }
\end{aligned}
$$


Proof. We will prove the lemma for $u \wedge k$. The proof for $u \vee k$ is analogous. Set

$$
\sigma_{n}(t)= \begin{cases}1, & \text { if } t \leq k \\ n(k-t)+1, & \text { if } t \in\left(k, k+\frac{1}{n}\right) \\ 0, & \text { if } t \geq k+\frac{1}{n}\end{cases}
$$

and $\theta_{n}(t)=\int_{0}^{t} \sigma_{n}(r) \mathrm{d} r, t \in \mathbb{R}$. Standard arguments show that $\theta_{n}(u) \rightarrow u \wedge k$ in $F M$ and, by the use of Lemma 5.3, that $\nabla_{\mathbb{X}} \theta_{n}(u)=\sigma_{n}(u) \nabla_{\mathbb{X}} u \rightarrow \mathbf{1}_{(-\infty, k)}(u) \nabla_{\mathbb{X}} u$ in $F M$. Hence, $u \wedge k \in W^{0,1}\left(\mathbb{X}^{D_{T}}\right)$ and $\nabla_{\mathbb{X}}(u \wedge k)=\mathbf{1}_{(-\infty, k)}(u) \nabla_{\mathbb{X}} u$. If we repeat the above arguments with $\sigma_{n}$ replaced by $\hat{\sigma}_{n}$ defined as

$$
\hat{\sigma}_{n}(t)= \begin{cases}1 & \text { if } t \leq k-\frac{1}{n} \\ n\left(k-\frac{1}{n}-t\right)+1 & \text { if } t \in\left(k-\frac{1}{n}, k\right), \\ 0 & \text { if } t \geq k\end{cases}
$$

we will obtain $\nabla_{\mathbb{X}}(u \wedge k)=\mathbf{1}_{(-\infty, k]}(u) \nabla_{\mathbb{X}} u$.

Let $F B$ denote the space of Borel measurable functions on $D_{T}$ such that for q.e. $(s, x) \in D_{T}, P_{s, x}^{\prime}\left(\operatorname{ess~sup}_{r \in\left[0, \zeta_{\tau}\right]}\left|u\left(\mathbf{X}_{r}\right)\right|<\infty\right)=1$. Observe that every quasi-càdlàg function belongs to $F B$.

One of the reason why the space $\mathcal{T}_{2}^{0,1}$ has been introduced is that the standard Sobolev space $L^{2}\left(0, T ; H_{0}^{1}(D)\right)$ lacks the property that $u \in L^{2}\left(0, T ; H_{0}^{1}(D)\right)$ if $u$ is quasi-càdlàg (natural class for solutions of equations with measure data) and $T_{k}(u) \in L^{2}\left(0, T ; H_{0}^{1}(D)\right)$ for every $k \geq 0$. The following lemma shows the stochastic Sobolev space has this remarkable feature.

LEMMA 5.5. If $u \in F B$ and $T_{k}(u) \in W^{0,1}\left(\mathbb{X}^{D_{T}}\right)$ for every $k \geq 0$, then $u \in$ $W^{0,1}\left(\mathbb{X}^{D_{T}}\right)$.

Proof. First, observe that for q.e. $(s, x) \in D_{T}$ and $\varepsilon>0$,

$$
P_{s, x}^{\prime}\left(\int_{0}^{\zeta_{\tau}}\left|T_{k}(u)-u\right|^{2}\left(\mathbf{X}_{r}\right) \mathrm{d} r>\varepsilon\right) \leq P_{s, x}^{\prime}\left(2 \int_{0}^{\zeta_{\tau}}|u|^{2} \mathbf{1}_{\{|u|>k\}}\left(\mathbf{X}_{r}\right) \mathrm{d} r>\varepsilon\right) \rightarrow 0,
$$

which shows that $T_{k}(u) \rightarrow u$ in $F M$. By Lemma 5.4, for $k<l$, we have

$$
\begin{aligned}
& P_{s, x}^{\prime}\left(\int_{0}^{\zeta_{\tau}}\left|\nabla_{\mathbb{X}} T_{k}(u)-\nabla_{\mathbb{X}} T_{l}(u)\right|^{2}\left(\mathbf{X}_{r}\right) \mathrm{d} r>\varepsilon\right) \\
& \quad=P_{s, x}^{\prime}\left(\int_{0}^{\zeta_{\tau}}\left|\nabla_{\mathbb{X}} T_{k}\left(T_{l}(u)\right)-\nabla_{\mathbb{X}} T_{l}(u)\right|^{2}\left(\mathbf{X}_{r}\right) \mathrm{d} r>\varepsilon\right) \\
& =P_{s, x}^{\prime}\left(\int_{0}^{\zeta_{\tau}}\left|\nabla_{\mathbb{X}} T_{l}(u) \mathbf{1}_{\left\{\left|T_{l}(u)\right|<k\right\}}-\nabla_{\mathbb{X}} T_{l}(u)\right|^{2}\left(\mathbf{X}_{r}\right) \mathrm{d} r>\varepsilon\right) \\
& =P_{s, x}^{\prime}\left(\int_{0}^{\zeta_{\tau}}\left|\nabla_{\mathbb{X}} T_{l}(u)\right|^{2} \mathbf{1}_{\{|u| \geq k\}}\left(\mathbf{X}_{r}\right) \mathrm{d} r>\varepsilon\right) .
\end{aligned}
$$

Observe that

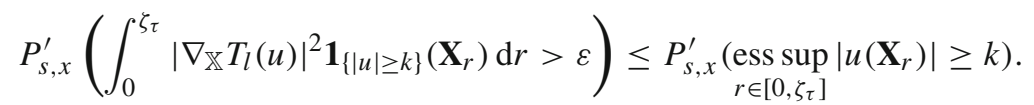


By the assumption that $u \in F B$, the right-hand side of the above inequality tends to zero as $k \rightarrow \infty$, which shows that $\nabla_{\mathbb{X}} T_{k}(u)-\nabla_{\mathbb{X}} T_{l}(u) \rightarrow 0$ in $F M$ as $k, l \rightarrow \infty$. Consequently, $u \in W^{0,1}\left(\mathbb{X}^{D_{T}}\right)$.

In [14], it is shown by examples that in general neither $\mathcal{T}_{2}^{0,1} \subset W^{0,1}\left(\mathbb{X}^{D_{T}}\right)$ nor $\mathcal{T}_{2}^{0,1} \supset W^{0,1}\left(\mathbb{X}^{D_{T}}\right)$. However, we have the following corollary to Lemma 5.5.

COROLLARY 5.6. If $u \in \mathcal{T}_{2}^{0,1}$ and $u \in F B$, then $u \in W^{0,1}\left(\mathbb{X}^{D_{T}}\right)$.

Besides being nonlinear, another drawback to the space $\mathcal{T}_{2}^{0,1}$ is that it is sometimes too small in practice. For instance, in [14], it is proved that solutions of some types of the obstacle problem are quasi-continuous and belong to $W^{0,1}\left(\mathbb{X}^{D_{T}}\right)$ but do not belong to $\mathcal{T}_{2}^{0,1}$.

EXAMPLE 5.7. Let $B\left(0, k^{-1}\right)=\left\{x \in \mathbb{R}^{2}:|x|<k^{-1}\right\}, D=B(0,1)$, and let $\alpha \in \mathbb{R}$.

(i) Put $v(t, x)=1-|x|^{-\alpha}$ for $(t, x) \in D_{T}$. Since $\operatorname{cap}_{N}\left(B\left(0, k^{-1}\right)\right) \rightarrow 0$ as $k \rightarrow \infty, v \in W^{0,1}\left(\mathbb{X}^{D_{T}}\right)$ and

$$
\nabla_{\mathbb{X}} v(t, x)=-\alpha x|x|^{-(\alpha+2)}, \quad(t, x) \in D_{T}
$$

for every $\alpha \in \mathbb{R}$. If $\alpha \geq 2$, then $v$ is not locally integrable, so there is no sense to speak about its distributional derivative. But let us observe that $v \in \mathcal{T}_{2}^{0,1}$ for every $\alpha \in \mathbb{R}$.

(ii) Put $u(t, x)=\sin v(t, x),(t, x) \in D_{T}$. Then, by Lemma 5.3, $u \in W^{0,1}\left(\mathbb{X}^{D_{T}}\right)$ and

$$
\nabla_{\mathbb{X}} u(t, x)=\alpha x|x|^{-(\alpha+2)} \cos \left(1-|x|^{-\alpha}\right)
$$

for every $\alpha \in \mathbb{R}$. One can check that for every $\varepsilon>0$ and $\alpha>1$,

$$
\int_{B(0, \varepsilon)} \alpha|x|^{-(\alpha+1)}\left|\cos \left(1-|x|^{-\alpha}\right)\right| \mathrm{d} x=\infty,
$$

which shows that if $\alpha>1$, then $u \notin \mathcal{T}_{2}^{0,1}$ since $T_{k}(u)=u$ for $k \geq 1$. However, $u \in L^{1}\left(D_{T}\right)$ and one can check that its distributional derivative is given by the formula

$$
\nabla u=\text { p.v. }\left(\alpha x|x|^{-(\alpha+2)} \cos \left(1-|x|^{-\alpha}\right)\right) \text {, }
$$

i.e., for every $\eta \in C_{0}^{\infty}(D)$,

$$
(\nabla u)(\eta)=\lim _{k \rightarrow \infty} \int_{D \backslash B\left(0, k^{-1}\right)} \alpha x|x|^{-(\alpha+2)} \cos \left(1-|x|^{-\alpha}\right) \eta(x) \mathrm{d} m(x) .
$$

Accordingly, even if the distributional derivative of $u \in W^{0,1}\left(\mathbb{X}^{D_{T}}\right)$ exists, it is not a function in general. 
Following [18], we adopt the following definition.

DEFINITION. We say that a measurable function $f: D_{T} \rightarrow \mathbb{R}$ is quasi-integrable if the function $\left[0, \zeta_{\tau}\right] \ni t \mapsto f\left(\mathbf{X}_{t}\right)$ belongs to $L^{1}\left(\left[0, \zeta_{\tau}\right]\right) P_{S, x}^{\prime}$-a.s. for q.e. $(s, x) \in$ $D_{T}$. The set of all quasi-integrable functions on $D_{T}$ will be denoted by $q L^{1}\left(D_{T}\right)$.

We say that a measurable function $u$ on $D_{T}$ is of class (FD) if for q.e. $(s, x) \in D_{T}$, the process $u(\mathbf{X})$ on $\left[0, \zeta_{\tau}\right]$ is of class (D) under the measure $P_{s, x}^{\prime}$.

REMARK 5.8. It is known (see [19]) that if $f$ is quasi-integrable in the analytic sense, i.e., if for every $\varepsilon>0$, there exists an open set $G_{\varepsilon} \subset D_{T}$ such that $\operatorname{cap}\left(G_{\varepsilon}\right)<\varepsilon$ and $f_{\mid D_{T} \backslash G_{\varepsilon}} \in L^{1}\left(D_{T} \backslash G_{\varepsilon}\right)$ then $f \in q L^{1}$. In particular, it follows that if $f \in L^{1}\left(D_{T}\right)$, then $f \in q L^{1}\left(D_{T}\right)$. It is also clear that if $R^{0, T}|f|<\infty, m_{1}$-a.e., then $f \in q L^{1}\left(D_{T}\right)$.

DEFINITION. We say that a measurable function $u: D_{T} \rightarrow \mathbb{R}^{N}$ is a solution of system (5.1) if

(a) $(t, x) \mapsto f(t, x, u(t, x)) \in q L^{1}\left(D_{T}\right), u \in W^{0,1}\left(\mathbb{X}^{D_{T}}\right)$ and $u$ is of class (FD),

(b) For q.e. $(s, x) \in D_{T}$,

$$
\begin{aligned}
u\left(\mathbf{X}_{t}\right)= & \mathbf{1}_{\left\{\zeta>T_{\tau}\right\}} \varphi\left(\mathbf{X}_{T_{\tau}}\right)+\int_{t}^{\zeta_{\tau}} f\left(\mathbf{X}_{r}, u\left(\mathbf{X}_{r}\right)\right) \mathrm{d} r \\
& +\int_{t}^{\zeta_{\tau}} \mathrm{d} A_{r}^{\mu}-\int_{t}^{\zeta_{\tau}} \sigma \nabla_{\mathbb{X}} u\left(\mathbf{X}_{r}\right) \mathrm{d} B_{r}, \quad t \in\left[0, \zeta_{\tau}\right], \quad P_{s, x}^{\prime} \text {-a.s. }
\end{aligned}
$$

In what follows for a given function $u$ on $D_{T}$, we set

$$
\bar{u}(t, x)=u(T-t, x), \quad(t, x) \in D_{T},
$$

and given $\mu \in \mathcal{M}_{0, b}\left(D_{T}\right)$, we denote by $\bar{\mu}$ the unique measure in $\mathcal{M}_{0, b}\left(D_{T}\right)$ such that

$$
\int_{D_{T}} \bar{\eta} \mathrm{d} \mu=\int_{D_{T}} \eta \mathrm{d} \bar{\mu}, \quad \eta \in \mathcal{B}_{b}\left(D_{T}\right) .
$$

DEFINITION. We say that $u$ is a solution of $(1.1)$ on $[0, T]$ if $\bar{u}$ is a solution of (5.1) with $f_{u}$ replaced by $\bar{f}_{u}, \mu$ replaced by $\bar{\mu}$ and $a$ replaced by $\bar{a}$.

DEFINITION. Let $(s, x) \in D_{T}$. We say that a pair $\left(Y^{s, x}, Z^{s, x}\right)$ consisting of an $\mathbb{R}^{N}$-valued process $Y^{s, x}$ and an $\mathbb{R}^{d} \times \mathbb{R}^{N}$-valued process $Z^{s, x}$ is a solution of $\operatorname{BSDE}_{s, x}(\varphi, D, f+\mathrm{d} \mu)$ if $Y^{s, x}, Z^{s, x}$ are $\left\{\mathcal{F}_{t}^{\prime}\right\}$ progressively measurable, $Y^{s, x}$ is càdlàg, $t \mapsto f\left(\mathbf{X}_{t}, Y_{t}^{s, x}, Z_{t}^{s, x}\right) \in L^{1}\left(0, \zeta_{\tau}\right), P_{s, x}^{\prime}$-a.s., $P_{s, x}^{\prime}\left(\int_{0}^{\zeta_{\tau}}\left|Z_{r}^{s, x}\right|^{2} \mathrm{~d} r<\infty\right)=1$ and

$$
\begin{aligned}
Y_{t}^{s, x}= & \mathbf{1}_{\left\{\zeta>T_{\tau}\right\}} \varphi\left(\mathbf{X}_{T_{\tau}}\right)+\int_{t}^{\zeta_{\tau}} f\left(\mathbf{X}_{r}, Y_{r}^{s, x}, Z_{r}^{s, x}\right) \mathrm{d} r \\
& +\int_{t}^{\zeta_{\tau}} \mathrm{d} A_{r}^{\mu}-\int_{t}^{\zeta_{\tau}} Z_{r}^{s, x} \mathrm{~d} B_{r}, \quad t \in\left[0, \zeta_{\tau}\right], \quad P_{s, x}^{\prime} \text {-a.s. }
\end{aligned}
$$


Observe that from the above two definitions, it follows that if $u$ is a solution of (5.1), then for q.e. $(s, x) \in D_{T}$ the pair $\left(Y^{s, x}, Z^{s, x}\right)=(Y, Z)$, where

$$
\left(Y_{t}, Z_{t}\right)=\left(u\left(\mathbf{X}_{t}\right), \sigma \nabla_{\mathbb{X}} u\left(\mathbf{X}_{t}\right)\right), \quad t \in\left[0, \zeta_{\tau}\right]
$$

is a solution of $\operatorname{BSDE}_{s, x}(\varphi, D, f+\mathrm{d} \mu)$. In the rest of this section, we are going to prove that under (H1)-(H4), this statement can be reversed in the following sense. For q.e. $(s, x) \in D_{T}$, there exists a unique solution $\left(Y^{s, x}, Z^{s, x}\right)$ of $\operatorname{BSDE}_{s, x}(\varphi, D, f+\mathrm{d} \mu)$ and if we set $u(s, x)=E_{s, x}^{\prime} Y_{0}^{s, x}$ for $(s, x) \in D_{T}$, then $u$ is a solution of (5.1). Moreover,

$$
Y_{t}^{s, x}=u\left(\mathbf{X}_{t}\right), t \in\left[0, \zeta_{\tau}\right], P_{s, x}^{\prime} \text {-a.s., } \quad Z^{s, x}=\sigma \nabla_{\mathbb{X}} u(\mathbf{X}), \mathrm{d} t \otimes P_{s, x}^{\prime} \text {-a.s. }
$$

Let us first state the following corollary to Theorem 3.7.

PROPOSITION 5.9. Assume (H1)-(H4). Let $F$ be the set of those $(s, x) \in D_{T}$ for which the data $\zeta_{\tau}, f(\mathbf{X}, \cdot, \cdot), \varphi\left(\mathbf{X}_{T_{\tau}}\right) \mathbf{1}_{\left\{\zeta>T_{\tau}\right\}}, A^{\mu}$ satisfy assumptions (A1)-(A4) under the measure $P_{s, x}^{\prime}$. Then, $\operatorname{cap}\left(D_{T} \backslash F\right)=0$, and for every $(s, x) \in F$, there exists a unique solution $\left(Y^{s, x}, Z^{s, x}\right)$ of $\operatorname{BSDE}_{s, x}(\varphi, D, f+\mathrm{d} \mu)$ such that $\left(Y^{s, x}, Z^{s, x}\right) \in$ $\mathcal{D}^{q} \otimes M^{q}$ for $q \in(0,1)$ and $Y^{s, x}$ is of class (D).

Proof. That $\operatorname{cap}\left(D_{T} \backslash F\right)=0$ follows from [16, Corollary 3.4] (see also [13, Remark 3.2]). The second assertion follows from Theorem 3.7.

The crucial issue in the proof of representation (5.8) and existence of solution to system (5.1) is regularity of the function $D_{T} \ni(s, x) \mapsto u(s, x)=E_{s, x}^{\prime} Y_{0}^{s, x}$. In most papers concerning probabilistic solutions for PDEs or stochastic representation for solutions of PDEs, the regularity is proved by using the results of probabilistic potential theory which may be applied when $u(s, x)=E_{s, x}^{\prime} \int_{0}^{\zeta_{\tau}} \mathrm{d} A_{r}$ for some AF of $\mathbb{X}^{\prime}$. Here, such approach cannot be applied because in general, $u$ does not admit the last representation [in general, integrals on the right-hand side of (1.8) do not exists]. We cope with the problem of regularity of $u$ in Propositions 5.10, 5.11.

PROPOSITION 5.10. Let $F$ be a Borel subset of $D_{T}$ such that $\operatorname{cap}\left(D_{T} \backslash F\right)=0$. Assume that for every $(s, x) \in F$, the real process $Y^{s, x}$ is a continuous semimartingale under $P_{s, x}^{\prime}$ such that $Y_{t \vee \zeta}^{s, x}=0, t \geq 0$, and there exists a Borel function $v$ on $D_{T}$ such that for every $(s, x) \in F$ and every $t \in\left[0, T_{\tau}\right]$,

$$
v\left(\mathbf{X}_{t}\right)=Y_{t}^{s, x}, \quad P_{s, x}^{\prime} \text {-a.s. }
$$

Then, $u(s, x)=E_{s, x}^{\prime} Y_{0}^{s, x}$ is a quasi-continuous version of $v$ and for every $(s, x) \in F$,

$$
u\left(\mathbf{X}_{t}\right)=Y_{t}^{s, x}, \quad t \in\left[0, \zeta_{\tau}\right], \quad P_{s, x}^{\prime} \text {-a.s. }
$$

Proof. Let $(s, x) \in F$. Since $Y^{s, x}$ is a continuous semimartingale, there exists a finite variation continuous process $R^{s, x}$ and $Z^{s, x} \in M$ such that

$$
Y_{t}^{s, x}=\mathbf{1}_{\left\{\zeta>T_{\tau}\right\}} v\left(\mathbf{X}_{T_{\tau}}\right)+\int_{t}^{\zeta_{\tau}} \mathrm{d} R_{r}^{s, x}-\int_{t}^{\zeta_{\tau}} Z_{r}^{s, x} \mathrm{~d} B_{r}, \quad t \in\left[0, \zeta_{\tau}\right], \quad P_{s, x}^{\prime} \text {-a.s. }
$$


First, let us assume additionally that $Y^{s, x}$ is bounded. Write $L^{n}=Y^{s, x}-\frac{1}{n}$ and $U=Y^{s, x}$. Then, $L^{n}<U$ and by [10], for every $(s, x) \in F$, there exists a unique solution $\left(Y^{n}, Z^{n}, R^{n}\right)$, on the space $\left(\Omega^{\prime}, \mathcal{F}_{\infty}^{\prime}, P_{s, x}^{\prime}\right)$, of the reflected BSDE with final condition $\xi \equiv v\left(\mathbf{X}_{T_{\tau}}^{D}\right)$, lower barrier $L^{n}$ and upper barrier $U\left(\operatorname{RBSDE}_{s, x}\left(\xi, 0, L^{n}, U\right)\right.$ for short) such that $Y^{n} \in \mathcal{S}^{2}, Z^{n} \in M$ and $R^{n} \in \mathcal{V}$. By [10, Theorem 1.3], $Y_{t}^{n} \leq$ $Y_{t}^{n+1}, t \in\left[0, \zeta_{\tau}\right], P_{s, x}^{\prime}$-a.s. for every $n \geq 1$. Therefore,

$$
Y_{t}^{n} \nearrow Y_{t}, \quad t \in\left[0, \zeta_{\tau}\right], \quad P_{s, x}^{\prime} \text {-a.s. }
$$

Let us fix $n \in \mathbb{N}$ and set $H_{t}=Y_{t}^{n}, t \in\left[0, \zeta_{\tau}\right]$. By [10],

$$
H_{t}^{k} \nearrow H_{t}, \quad t \in\left[0, \zeta_{\tau}\right], \quad P_{s, x}^{\prime} \text {-a.s., }
$$

where $H^{k}$ is the first component of the solution of $\operatorname{RBSDE}_{s, x}\left(0, f_{k}, U\right)$ with $f_{k}(t, y)=$ $k\left(y-L_{t}^{n}\right)^{-}$. Let $H^{k, l}$ denote the first component of the solution of $\operatorname{BSDE}_{s, x}(v(T, \cdot)$, $\left.f_{k, l}\right)$ with $f_{k, l}(t, y)=k\left(y-L_{t}^{n}\right)^{-}-l\left(y-U_{t}\right)^{+}$. Then, by [7],

$$
H_{t}^{k, l} \searrow H_{t}^{k}, \quad t \in\left[0, \zeta_{\tau}\right], \quad P_{s, x}^{\prime} \text {-a.s. }
$$

By (5.9), $f_{k, l}(\cdot, y)=k\left(y-v(\mathbf{X})+\frac{1}{n}\right)^{-}-l(y-v(\mathbf{X}))^{+}, \mathrm{d} t \otimes P_{s, x^{\prime}}^{\prime}$ a.e. on $\left[0, \zeta_{\tau}\right] \times \Omega$ for every $y \in \mathbb{R}$. Set $g_{k, l}(t, x, y)=k\left(y-v(x)+\frac{1}{n}\right)^{-}-l(y-v(x))^{+}$. By [13],

$$
H_{t}^{k, l}=h_{k, l}\left(\mathbf{X}_{t}\right), \quad t \in\left[0, \zeta_{\tau}\right], \quad P_{s, x}^{\prime} \text {-a.s., }
$$

where $h_{k, l}$ is a quasi-continuous version of the solution of $\operatorname{PDE}\left(0, g_{k, l}\right)$. Let us put

$$
h(s, x)=\limsup _{k \rightarrow \infty} \liminf _{l \rightarrow \infty} h_{k, l}(s, x), \quad(s, x) \in D_{T} .
$$

Then, by (5.11) and (5.12),

$$
H_{t}=h\left(\mathbf{X}_{t}\right), \quad t \in\left[0, \zeta_{\tau}\right], \quad P_{s, x}^{\prime} \text {-a.s. }
$$

for every $(s, x) \in F$. In particular, since $H \in \mathcal{S}^{2}$, the function $h$ is quasi-continuous. From what has already been proved it follows that for every $n \geq 1$, there exists a quasi-continuous function $u_{n}$ such that

$$
Y_{t}^{n}=u_{n}\left(\mathbf{X}_{t}\right), \quad t \in\left[0, \zeta_{\tau}\right], \quad P_{s, x}^{\prime} \text {-a.s. }
$$

for every $(s, x) \in F$. Putting $u(s, x)=\lim _{\sup } \rightarrow \infty u_{n}(s, x),(s, x) \in D_{T}$, we get by (5.10) the desired result.

Now, we show how to dispense with the assumption that $Y^{s, x}$ is bounded. By the Itô-Tanaka formula, $T_{k}(Y)$ is a semimartingale, so by the first part of the proof, the function $u_{k}$ defined as $u_{k}(s, x)=E_{s, x}^{\prime} T_{k}\left(Y_{0}^{s, x}\right),(s, x) \in D_{T}$, is quasi-continuous and for each $k \in \mathbb{N}$,

$$
T_{k}\left(Y_{t}^{s, x}\right)=u_{k}\left(\mathbf{X}_{t}\right), \quad t \in\left[0, \zeta_{\tau}\right], \quad P_{s, x}^{\prime} \text {-a.s. }
$$

for every $(s, x) \in F$. But $u_{k}(s, x)=E_{s, x} T_{k}\left(Y_{0}^{s, x}\right)=T_{k}\left(E_{s, x} Y_{0}^{s, x}\right)$ since $Y_{0}^{s, x}$ is constant $P_{s, x}^{\prime}$-a.s. Therefore, letting $k \rightarrow \infty$ in (5.13), we get the desired result for $Y$. 
PROPOSITION 5.11. Let $F$ be a Borel subset of $D_{T}$ such that $\operatorname{cap}\left(D_{T} \backslash F\right)=0$ and let $u: D_{T} \rightarrow \mathbb{R}$ be a Borel function. If $u(\mathbf{X})$ is a continuous semimartingale under $P_{s, x}^{\prime}$ on $\left[0, \zeta_{\tau}\right]$ for $(s, x) \in F$, then $u \in W^{0,1}\left(\mathbb{X}^{D_{T}}\right)$ and there exists $C A F$ A of finite variation such that

$$
u\left(\mathbf{X}_{t}\right)=u(s, x)+\int_{0}^{t} \mathrm{~d} A_{r}+\int_{0}^{t} \sigma \nabla_{\mathbb{X}} u\left(\mathbf{X}_{r}\right) \mathrm{d} B_{r}, \quad t \in\left[0, \zeta_{\tau}\right], \quad P_{s, x}^{\prime} \text {-a.s. }
$$

for every $(s, x) \in F$.

Proof. We adopt the notation of the proof of Proposition 5.10 and put $M_{t}^{n}=\int_{0}^{t} Z_{r}^{n} \mathrm{~d} B_{r}$. Let us first assume that $u$ is bounded. By [14], we know that

$$
Y_{t}^{n}=u_{n}\left(\mathbf{X}_{t}\right), t \in\left[0, \zeta_{\tau}\right], \quad \sigma \nabla_{\mathbb{X}} u_{n}(\mathbf{X})=Z^{n}, \mathrm{~d} t \otimes P_{s, x}^{\prime} \text {-a.e. },
$$

where $u_{n} \in W^{0,1}\left(\mathbb{X}^{D_{T}}\right)$ is a solution of OP $\left(v(T, \cdot), 0, L^{n}, U\right)$ (with two barriers). Fix $(s, x) \in F$. Since $Y=u(\mathbf{X})$ is a continuous semimartingale and the underlying filtration is Brownian, there exists a finite variation continuous process $R^{s, x}$ and a process $Z^{s, x} \in M$ such that

$$
Y_{t}=Y_{0}+\int_{0}^{t} \mathrm{~d} R_{r}^{s, x}+\int_{0}^{t} Z_{r}^{s, x} \mathrm{~d} B_{r}, \quad t \in\left[0, \zeta_{\tau}\right]
$$

Put $M_{t}^{s, x}=\int_{0}^{t} Z_{r}^{s, x} \mathrm{~d} B_{r}$. Since the process $Y$ is continuous, from the proof of Proposition 5.10 and Dini's theorem, it follows that

$$
P_{s, x}^{\prime}\left(\sup _{t \in\left[0, \zeta_{\tau}\right]}\left|Y_{t}^{n}-Y_{t}\right|^{2}>\varepsilon\right) \rightarrow 0
$$

Moreover, by [14, Proposition 6.1],

$$
\mathrm{d} R^{n,+} \leq \mathbf{1}_{\left\{Y_{t}^{n}=L_{t}^{n}\right\}} \mathrm{d} R_{t}^{+}, \quad \mathrm{d} R^{n,-} \leq \mathbf{1}_{\left\{Y_{t}^{n}=U_{t}\right\}} \mathrm{d} R_{t}^{-} .
$$

Therefore, there exists a sequence of stopping times $\left\{\tau_{k}\right\}$ such that $\tau_{k} \leq \tau_{k+1}, k \geq$ $1, \tau_{k} \rightarrow \zeta_{\tau} P_{s, x}^{\prime}$-a.s. for q.e. $(s, x) \in D_{T}$ and for every $k \geq 1$, the sequence $\left\{Y^{n, \tau_{k}}\right\}$ satisfies the condition UT (see, e.g., [33]). Therefore,

$$
P_{s, x}^{\prime}\left(\left\langle M^{n}-M^{s, x}\right\rangle_{\zeta_{\tau}}>\varepsilon\right) \rightarrow 0
$$

(see, e.g., [33, Proposition 1.5]). From (5.14)-(5.17), we deduce that $u \in W^{0,1}\left(\mathbb{X}^{D_{T}}\right)$ and $\sigma \nabla_{\mathbb{X}} u\left(\mathbf{X}^{D}\right)=Z^{s, x}, \mathrm{~d} t \otimes P_{s, x}^{\prime}$-a.e. Putting

$$
A_{t}=u\left(\mathbf{X}_{t}\right)-u(s, x)-\int_{0}^{t} \sigma \nabla_{\mathbb{X}} u\left(\mathbf{X}_{r}\right) \mathrm{d} B_{r}
$$

we see that $A$ is a CAF of finite variation and $P_{s, x}^{\prime}\left(R_{t}^{s, x}=A_{t}, t \in\left[0, \zeta_{\tau}\right]\right)=1$, which proves the proposition in the case $u$ is bounded. In the general case, (5.15) still holds. By the Itô-Tanaka formula, for every $k>0$, we have 


$$
\begin{aligned}
T_{k}(u)\left(\mathbf{X}_{t}\right)= & T_{k}(u(s, x))+\int_{0}^{t} \mathbf{1}_{(-k, k]}\left(u\left(\mathbf{X}_{r}\right)\right) \mathrm{d} R_{r}^{s, x} \\
& +\int_{0}^{t} \mathbf{1}_{(-k, k]}\left(u\left(\mathbf{X}_{r}\right)\right) Z_{r}^{s, x} \mathrm{~d} B_{r}+\frac{1}{2}\left(L_{t}^{k}-L_{t}^{-k}\right), \quad t \in\left[0, \zeta_{\tau}\right],
\end{aligned}
$$

where $L^{k}$ (respectively, $L^{-k}$ ) is the local time of the process $u(\mathbf{X})$ at $k$ (respectively, $-k)$. By the first step of the proof, $T_{k}(u) \in W^{0,1}\left(\mathbb{X}^{D_{T}}\right)$ for every $k \geq 0$. Since $u$ is quasi-continuous, $u \in F B$ and hence, by Lemma 5.5, $u \in W^{0,1}\left(\mathbb{X}^{D_{T}}\right)$. By the first step of the proof,

$$
Z^{s, x} \mathbf{1}_{(-k, k]}(u(\mathbf{X}))=\sigma \nabla_{\mathbb{X}}\left(T_{k}(u)\right)(\mathbf{X}), \quad \mathrm{d} t \otimes P_{s, x}^{\prime} \text {-a.e. }
$$

Hence, by Lemma 5.4,

$$
Z^{s, x} \mathbf{1}_{(-k, k]}(u(\mathbf{X}))=\mathbf{1}_{(-k, k]}(u(\mathbf{X})) \sigma \nabla_{\mathbb{X}} u(\mathbf{X}), \quad \mathrm{d} t \otimes P_{s, x}^{\prime} \text {-a.e. }
$$

for every $k \geq 0$, which implies that

$$
Z^{s, x}=\sigma \nabla_{\mathbb{X}} u(\mathbf{X}), \quad \mathrm{d} t \otimes P_{s, x}^{\prime} \text {-a.e. }
$$

The rest of the proof runs as in the first step.

THEOREM 5.12. Assume (H1)-(H4). Then, there exists a unique solution $u$ of system (5.1). Moreover, there exists a version of $u$ (still denoted by $u$ ) such that (5.7) is satisfied for every $(s, x) \in F$, where $F$ is defined in Corollary 5.9.

Proof. By Proposition 5.9, for every $(s, x) \in F$, there exists a solution $\left(Y^{s, x}, Z^{s, x}\right)$ of $\operatorname{BSDE}_{s, x}(\varphi, D, f+\mathrm{d} \mu)$ such that $\left(Y^{s, x}, Z^{s, x}\right) \in \mathcal{D}^{q} \otimes M^{q}$ for $q \in(0,1)$ and $Y^{s, x}$ is of class (D). By [19, Proposition 3.5], $u\left(\mathbf{X}_{t}\right)=Y_{t}^{s, x}, P_{s, x}^{\prime}$-a.s. for every $(s, x) \in F$ and every $t \in\left[0, T_{\tau}\right]$, where $u(s, x)=E_{s, x}^{\prime} Y_{0}^{s, x}$. Our aim is to show that $u$ is quasi-càdlàg, belongs to $W^{0,1}\left(\mathbb{X}^{D_{T}}\right)$ and representation (5.8) holds q.e. Note that we cannot apply directly Propositions 5.10, 5.11 because we do not know that $u(\mathbf{X})$ is continuous. To overcome the difficulty, let us consider a solution $\left(Y^{1, s, x}, Z^{1, s, x}\right) \in \mathcal{D}^{q} \otimes M^{q}, q \in$ $(0,1)$, of the $\operatorname{BSDE}_{s, x}(\varphi, D, \mu)$ such that $Y^{1, s, x}$ is of class (D). By [19, Proposition 3.7], $u_{1}(s, x)=E_{s, x}^{\prime} Y_{0}^{1, s, x}$ is quasi-càdlàg, $u_{1} \in \mathcal{T}_{2}^{0,1}$ and for every $(s, x) \in F$,

$$
u_{1}\left(\mathbf{X}_{t}\right)=Y_{t}^{1, s, x}, \quad t \in\left[0, \zeta_{\tau}\right], P_{s, x}^{\prime} \text {-a.s., } \quad \sigma \nabla u_{1}(\mathbf{X})=Z^{1, s, x}, \quad \mathrm{~d} t \otimes P_{s, x}^{\prime} \text {-a.e. }
$$

Observe that

$$
Y_{t}^{s, x}-Y_{t}^{1, s, x}=\int_{t}^{\zeta_{\tau}} f\left(\mathbf{X}_{r}, Y_{r}^{s, x}\right) \mathrm{d} r+\int_{t}^{\zeta_{\tau}}\left(Z_{r}^{s, x}-Z_{r}^{1, s, x}\right) \mathrm{d} B_{r}, t \in\left[0, \zeta_{\tau}\right], P_{s, x}^{\prime} \text {-a.s. }
$$

Applying Proposition 5.10 to each coordinate of the process $Y^{s, x}-Y^{1, s, x}$, we conclude that there is a quasi-continuous function $v: D_{T} \rightarrow \mathbb{R}^{N}$ such that for every $(s, x) \in F$,

$$
Y_{t}^{s, x}-Y_{t}^{1, s, x}=v\left(\mathbf{X}_{t}\right), \quad t \in\left[0, \zeta_{\tau}\right], \quad P_{s, x}^{\prime} \text {-a.s. }
$$


From this and (5.18),

$$
Y_{t}^{s, x}=u_{1}\left(\mathbf{X}_{t}\right)+v\left(\mathbf{X}_{t}\right), \quad t \in\left[0, \zeta_{\tau}\right], \quad P_{s, x}^{\prime} \text {-a.s. }
$$

But

$$
v(s, x)=E_{s, x}^{\prime} Y_{0}^{s, x}-E_{s, x}^{\prime} Y_{0}^{1, s, x}=u_{1}(s, x)-u(s, x)
$$

for q.e. $(s, x) \in D_{T}$. Therefore,

$$
Y_{t}^{s, x}=u\left(\mathbf{X}_{t}\right), \quad t \in\left[0, \zeta_{\tau}\right], \quad P_{s, x}^{\prime} \text {-a.s. }
$$

It follows in particular that $u$ is quasi-càdlàg. Since $u_{1}$ is quasi-càdlàg, it belongs to $F B$. Consequently, by Corollary 5.6, $u_{1} \in W^{0,1}\left(\mathbb{X}^{D_{T}}\right)$. Therefore, from (5.18) and Proposition 5.11 applied to each coordinate of the function $v$, it follows that $u \in W^{0,1}\left(\mathbb{X}^{D_{T}}\right)$ and

$$
\sigma \nabla_{\mathbb{X}} u(\mathbf{X})=Z^{s, x}, \quad \mathrm{~d} t \otimes P_{s, x^{-a}}^{\prime} \text { e. }
$$

for every $(s, x) \in F$. Thus, $u$ is a solution of (5.1). Uniqueness follows from Theorem 3.6.

PROPOSITION 5.13. Let $u$ be a solution of system (5.1). Then, $\nabla_{\mathbb{X}} u \in L_{l o c}^{q}\left(D_{T}\right)$ for every $q \in(0,1)$.

Proof. Since $u$ is of class (FD), $u(\mathbf{X})$ is of class (D) on $\left[0, \zeta_{\tau}\right]$ under $P_{s, x}^{\prime}$ for q.e. $(s, x) \in$ $D_{T}$. Therefore, $\left(u(\mathbf{X}), \sigma \nabla_{\mathbb{X}} u(\mathbf{X})\right)$ is a unique solution of $\operatorname{BSDE}_{s, x}(\varphi, D, f+\mathrm{d} \mu)$. By [3, Proposition 6.4], $u \in \mathcal{D}^{q}$ and $\nabla_{\mathbb{X}} u \in M^{q}$ for $q \in(0,1)$. Applying the Itô-Meyer formula to (5.7) and using (H2) and the fact that $u$ is of class (FD), we get

$$
\left|u\left(\mathbf{X}_{t}\right)\right| \leq E_{s, x}^{\prime}\left(\left|\varphi\left(\mathbf{X}_{T_{\tau}}\right)\right| \mathbf{1}_{\left\{\zeta_{\tau}>T_{\tau}\right\}}+\int_{0}^{\zeta_{\tau}}\left|f\left(\mathbf{X}_{r}, 0\right)\right| \mathrm{d} r+\int_{0}^{\zeta_{\tau}} \mathrm{d}\left|A^{\mu}\right|_{r} \mid \mathcal{F}_{t}^{\prime}\right) .
$$

By [3, Lemma 6.1], for every $q \in(0,1)$,

$$
\begin{aligned}
E_{s, x}^{\prime} \sup _{0 \leq t \leq \zeta_{\tau}}\left|u\left(\mathbf{X}_{t}\right)\right|^{q} \leq & (1-q)^{-1} E_{s, x}^{\prime}\left(1+\left|\varphi\left(\mathbf{X}_{T_{\tau}}\right)\right| \mathbf{1}_{\left\{\zeta_{\tau}>T_{\tau}\right\}}\right. \\
& \left.+\int_{0}^{\zeta_{\tau}}\left|f\left(\mathbf{X}_{r}, 0\right)\right| \mathrm{d} r+\int_{0}^{\zeta_{\tau}} \mathrm{d}\left|A^{\mu}\right|_{r}\right)
\end{aligned}
$$

By the above estimate and Lemma 3.1,

$$
\begin{aligned}
E_{s, x}^{\prime}\left(\int_{0}^{\zeta_{\tau}}\left|\sigma \nabla_{\mathbb{X}} u\left(\mathbf{X}_{r}\right)\right|^{2} \mathrm{~d} r\right)^{q / 2} \leq & c(q) E_{s, x}^{\prime}\left(1+\left|\varphi\left(\mathbf{X}_{T_{\tau}}\right)\right| \mathbf{1}_{\left\{\zeta_{\tau}>T_{\tau}\right\}}\right. \\
& \left.+\int_{0}^{\zeta_{\tau}}\left|f\left(\mathbf{X}_{r}, 0\right)\right| \mathrm{d} r+\int_{0}^{\zeta_{\tau}} \mathrm{d}\left|A^{\mu}\right|_{r}\right)
\end{aligned}
$$


for every $q \in(0,1)$. Since $q \in(0,1)$, we have

$$
\begin{aligned}
E_{s, x}^{\prime}\left(\int_{0}^{\zeta_{\tau}}\left|\sigma \nabla_{\mathbb{X}} u\left(\mathbf{X}_{r}\right)\right|^{2} \mathrm{~d} r\right)^{q / 2} & \geq \Lambda^{-1} E_{s, x}^{\prime}\left(\int_{0}^{\zeta_{\tau}}\left|\nabla_{\mathbb{X}} u\left(\mathbf{X}_{r}\right)\right|^{q} \mathrm{~d} r \cdot \zeta_{\tau}^{q / 2-1}\right) \\
& \geq \Lambda^{-1} T^{q / 2-1} E_{s, x}^{\prime}\left(\int_{0}^{\zeta_{\tau}}\left|\nabla_{\mathbb{X}} u\left(\mathbf{X}_{r}\right)\right|^{q} \mathrm{~d} r\right)
\end{aligned}
$$

By the above,

$$
\iint_{D_{T}}\left|\nabla_{\mathbb{X}} u(r, y)\right|^{q} p_{D}(s, x, r, y) \mathrm{d} r d y=E_{s, x}^{\prime}\left(\int_{0}^{\zeta_{\tau}}\left|\nabla_{\mathbb{X}} u\left(\mathbf{X}_{r}\right)\right|^{q} \mathrm{~d} r\right)<\infty
$$

for q.e. $(s, x) \in D_{T}$, where $p_{D}$ is the transition density of the process $\mathbb{X}$ killed on exiting $D$. Therefore, the desired result follows from the well-known fact that $p_{D}(s, x, \cdot, \cdot)$ is continuous and strictly positive on $(s, T] \times D$ (see [1]).

REMARK 5.14. From [19], it follows that if $u$ is a probabilistic solution of (5.1) such that $f(\cdot, u) \in L^{1}\left(D_{T}\right)$, then $u \in \mathcal{T}_{2}^{0,1}, u \in L^{q}\left(0, T ; W_{0}^{1, q}(D)\right)$ for $q \in\left[1, \frac{d+2}{d+1}\right)$ and $u$ is a renormalized (entropy) solution of (5.1).

\section{Large-time asymptotics}

In this section, we prove our main results (1.10) and (1.11) on large-time behavior of solutions of (1.1) in case $A, \mu$ and $f$ do not depend on time. A key role in obtaining (1.10), (1.11) play some a priori estimates on solutions of BSDEs which we present below.

\subsection{Large-time estimates for the solutions of BSDEs}

Let $\mu$ be a soft measure such that $A^{\mu}$ is a CAF of $\mathbb{X}^{\prime}$. Let $\zeta_{\tau}^{n}=(n-\tau(0)) \wedge \zeta$ and let $\left(Y^{n}, Z^{n}\right)$ be a solution of the $\operatorname{BSDE}_{s, x}$

$$
\begin{aligned}
Y_{t}^{n}= & \varphi\left(\mathbf{X}_{n-\tau(0)}\right) \mathbf{1}_{\{\zeta>n-\tau(0)\}}+\int_{t}^{\zeta_{\tau}^{n}} f\left(\mathbf{X}_{r}, Y_{r}^{n}\right) \mathrm{d} r \\
& +\int_{t}^{\zeta_{\tau}^{n}} \mathrm{~d} A_{r}^{\mu}-\int_{t}^{\zeta_{\tau}^{n}} Z_{r}^{n} \mathrm{~d} B_{r}, \quad 0 \leq t \leq \zeta_{\tau}^{n}
\end{aligned}
$$

such that $\left(Y^{n}, Z^{n}\right) \in \mathcal{D}^{q} \otimes M^{q}$ for $q \in(0,1)$ and $Y^{n}$ is of class (D). In the remainder of the paper, we adopt the convention that $Y_{t}^{n}=0, t \geq n-\tau(0)$ and $Z_{r}^{n}=0$, $r \geq n-\tau(0)$. 
PROPOSITION 6.1. Assume that (H1)-(H4) are satisfied with $\alpha \leq 0$. Let $n<m$ and $\delta Y_{t}=Y_{t}^{m}-Y_{t}^{n}, \delta Z_{t}=Z_{t}^{m}-Z_{t}^{n}$. Then, for every $q \in(0,1)$,

$$
\begin{aligned}
& E_{s, x}^{\prime} \sup _{t \geq 0}\left|\delta Y_{t}\right|^{q}+\epsilon E_{s, x}^{\prime}\left(\int_{0}^{\zeta}\left|\delta Z_{r}\right|^{2} \mathrm{~d} r\right)^{q / 2} \\
& \leq(1-q)^{-1}\left(1+\epsilon 2 C_{q}\right) E_{s, x}^{\prime}\left(\left|\varphi\left(\mathbf{X}_{n-s}\right)\right|^{q} \mathbf{1}_{\{\zeta>n-s\}}+\left|\varphi\left(\mathbf{X}_{m-s}\right)\right|^{q} \mathbf{1}_{\{\zeta>m-s\}}\right. \\
& \left.\quad+\left(\int_{n}^{\zeta} \mathrm{d}\left|A^{\mu}\right|_{r}\right)^{q}+\left(\int_{n}^{\zeta}\left|f\left(\mathbf{X}_{r}, 0\right)\right| \mathrm{d} r\right)^{q}\right)
\end{aligned}
$$

for $\epsilon=0,1$, where $C_{q}$ is the constant from Lemma 3.1 .

Proof. Observe that

$Y_{t}^{n}=Y_{0}^{n}-\int_{0}^{t} \mathbf{1}_{\left[0, \zeta_{\tau}^{n}\right]}(r) f\left(\mathbf{X}_{r}, Y_{r}^{n}\right) \mathrm{d} r-\int_{0}^{t} \mathbf{1}_{\left[0, \zeta_{\tau}^{n}\right]}(r) \mathrm{d} A_{r}^{\mu}+\int_{0}^{t} d V_{r}^{n}+\int_{0}^{t} Z_{r}^{n} \mathrm{~d} B_{r}$

for $t \geq 0$, where

$$
V_{t}^{n}=\left\{\begin{array}{l}
0, \quad t<n-\tau(0) \\
-Y_{n-\tau(0)}^{n}, \quad t \geq n-\tau(0)
\end{array}\right.
$$

Put

$$
\begin{aligned}
\psi(t)= & -Y_{0}^{m}+\int_{0}^{t} \mathbf{1}_{\left[0, \zeta_{\tau}^{m}\right]}(r) f\left(\mathbf{X}_{r}, Y_{r}^{m}\right) \mathrm{d} r+\int_{0}^{t} \mathbf{1}_{\left[0, \zeta_{\tau}^{m}\right]}(r) \mathrm{d} A_{r}^{\mu}-\int_{0}^{t} d V_{r}^{m} \\
& +Y_{0}^{n}-\int_{0}^{t} \mathbf{1}_{\left[0, \zeta_{\tau}^{n}\right]}(r) f\left(\mathbf{X}_{r}, Y_{r}^{n}\right) \mathrm{d} r-\int_{0}^{t} \mathbf{1}_{\left[0, \zeta_{\tau}^{n}\right]}(r) \mathrm{d} A_{r}^{\mu}+\int_{0}^{t} d V_{r}^{n}, \quad t \geq 0 .
\end{aligned}
$$

By the Itô-Meyer formula (see Corollary 2.3) and standard localization procedure for local martingale $N=\int_{0}^{\cdot}\left\langle\operatorname{sg} \hat{g}\left(\delta Y_{r-}\right), \delta Z_{r} \mathrm{~d} B_{r}\right\rangle$, for $t \leq m-\tau(0)$, we have

$$
\left|\delta Y_{t}\right| \leq E_{s, x}^{\prime}\left(\left|\delta Y_{m-s}\right|+\int_{t}^{m-s}\left\langle\operatorname{sgn}\left(\delta Y_{r-}\right), \mathrm{d} \psi(r)\right\rangle \mid \mathcal{F}_{t}^{\prime}\right)
$$

Suppose that $n-\tau(0)<t \leq m-\tau(0)$. Since $\left|\delta Y_{m-\tau(0)}\right|=0$, it follows from (6.1) that

$$
\begin{aligned}
\left|\delta Y_{t}\right| \leq & E_{s, x}^{\prime}\left(\int_{t}^{m-s}\left\langle\operatorname{sg} \hat{g}\left(\delta Y_{r-}\right) \mathbf{1}_{\left[0, \zeta_{\tau}^{m}\right]}(r), f\left(\mathbf{X}_{r}, Y_{r}^{m}\right)\right\rangle \mathrm{d} r\right. \\
& \left.+\int_{t}^{m-s}\left\langle\mathbf{1}_{\left[0, \zeta_{\tau}^{m}\right]}(r) \operatorname{sg} n\left(\delta Y_{r-}\right), \mathrm{d} A_{r}^{\mu}\right\rangle-\int_{t}^{m-s}\left\langle\operatorname{sg} n\left(\delta Y_{r-}\right) \mathbf{1}_{\left[0, \zeta_{\tau}^{m}\right]}(r), d V_{r}^{m}\right\rangle \mid \mathcal{F}_{t}^{\prime}\right) .
\end{aligned}
$$

Moreover,

$$
\int_{t}^{\zeta_{\tau}^{m}}\left\langle\operatorname{sg} \hat{\operatorname{gn}}\left(\delta Y_{r-}\right), f\left(\mathbf{X}_{r}, Y_{r}^{m}\right)\right\rangle \mathrm{d} r=\int_{t}^{\zeta_{\tau}^{m}}\left\langle\operatorname{sg} \mathrm{g}\left(Y_{r-}^{m}\right), f\left(\mathbf{X}_{r}, Y_{r}^{m}\right)\right\rangle \mathrm{d} r \leq \int_{n-\tau(0)}^{\zeta}\left|f\left(\mathbf{X}_{r}, 0\right)\right| \mathrm{d} r
$$


and

$$
\begin{aligned}
\int_{t}^{\zeta_{\tau}^{m}}\left\langle\operatorname{sg} \mathrm{gn}\left(\delta Y_{r-}\right), \mathrm{d} A_{r}^{\mu}\right\rangle & \leq \int_{n-\tau(0)}^{\zeta} \mathrm{d}\left|A^{\mu}\right|_{r}, \int_{t}^{\zeta_{\tau}^{m}}\left\langle\operatorname{sg} \mathrm{gn}\left(\delta Y_{r-}\right), d V_{r}^{m}\right\rangle \\
& \leq\left|\varphi\left(\mathbf{X}_{m-\tau(0)}\right)\right| \mathbf{1}_{\left[0, \zeta_{\tau}^{m}\right]} .
\end{aligned}
$$

By the above estimates, if $n-\tau(0)<t \leq m-\tau(0)$, then

$$
\left|\delta Y_{t}\right| \leq E_{s, x}^{\prime}\left(\int_{n-s}^{\zeta} \mathrm{d}\left|A^{\mu}\right|_{r}+\int_{n-s}^{\zeta}\left|f\left(\mathbf{X}_{r}, 0\right)\right| \mathrm{d} r+\left|\varphi\left(\mathbf{X}_{m-s}\right)\right| \mathbf{1}_{\left[0, \zeta_{\tau}^{m}\right]} \mid \mathcal{F}_{t}^{\prime}\right) \equiv I(n, m) .
$$

Suppose now that $0 \leq t \leq n-\tau(0)$. Then, by (6.1),

$$
\begin{aligned}
& \left|\delta Y_{t}\right| \leq E_{s, x}^{\prime}\left(\int _ { t } ^ { m - s } \left\langle\mathbf{1}_{\left[0, \zeta_{\tau}^{m}\right]}(r) f\left(\mathbf{X}_{r}, Y_{r}^{m}\right)-\mathbf{1}_{\left[0, \zeta_{\tau}^{n}\right]}(r) f\left(\mathbf{X}_{r}, Y_{r}^{n}\right), \operatorname{sg} \mathrm{g}\left(\delta Y_{r}\right\rangle \mathrm{d} r\right.\right. \\
& +\int_{t}^{m-s}\left\langle\left(\mathbf{1}_{\left[0, \zeta_{\tau}^{m}\right]}(r)-\mathbf{1}_{\left[0, \zeta_{\tau}^{n}\right]}(r)\right) \operatorname{sg} \mathrm{g}\left(\delta Y_{r-}\right), \mathrm{d} A_{r}^{\mu}\right\rangle
\end{aligned}
$$

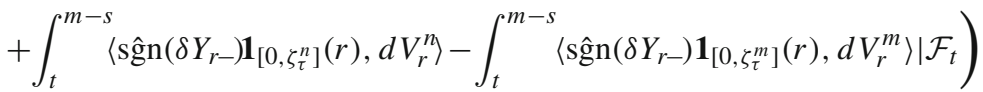

$$
\begin{aligned}
& \leq I(n, m)+E_{s, x}^{\prime}\left(\int_{t}^{n-s}\left\langle\mathbf{1}_{\left[0, \zeta_{\tau}^{m}\right]}(r) f\left(\mathbf{X}_{r}, Y_{r}^{m}\right)-\mathbf{1}_{\left[0, \zeta_{\tau}^{n}\right]}(r) f\left(\mathbf{X}_{r}, Y_{r}^{n}\right), \operatorname{sg} \hat{g}\left(\delta Y_{r}\right)\right\rangle \mathrm{d} r\right. \\
& +\int_{t}^{n-s}\left\langle\left(\mathbf{1}_{\left[0, \zeta_{\tau}^{m}\right]}(r)-\mathbf{1}_{\left[0, \zeta_{\tau}^{n}\right]}(r)\right) \operatorname{sg} \mathrm{g}\left(\delta Y_{r-}\right), \mathrm{d} A_{r}^{\mu}\right\rangle \\
& \left.+\int_{t}^{n-s}\left\langle\operatorname{sg} n\left(\delta Y_{r-}\right) \mathbf{1}_{\left[0, \zeta_{\tau}^{n}\right]}(r), d V_{r}^{n}\right\rangle-\int_{t}^{n-s}\left\langle\operatorname{sg} \hat{g}\left(\delta Y_{r-}\right) \mathbf{1}_{\left[0, \zeta_{\tau}^{m}\right]}(r), d V_{r}^{m}\right\rangle \mid \mathcal{F}_{t}^{\prime}\right) .
\end{aligned}
$$

The last term is equal to zero. By $(\mathrm{H} 2)$,

$$
\begin{gathered}
\left.\int_{t}^{n-\tau(0)}\left\langle\mathbf{1}_{\left[0, \zeta_{\tau}^{m}\right]}(r) f\left(\mathbf{X}_{r}, Y_{r}^{m}\right)-\mathbf{1}_{\left[0, \zeta_{\tau}^{n}\right]}(r) f\left(\mathbf{X}_{r}, Y_{r}^{n}\right)\right), \operatorname{sg} n\left(\delta Y_{r-}\right)\right\rangle \mathrm{d} r \\
=\int_{t}^{n-\tau(0)}\left\langle\mathbf{1}_{\left[0, \zeta_{\tau}^{n}\right]}(r)\left(f\left(\mathbf{X}_{r}, Y_{r}^{m}\right)-f\left(\mathbf{X}_{r}, Y_{r}^{n}\right)\right), \operatorname{sg} n\left(\delta Y_{r-}\right)\right\rangle \mathrm{d} r \leq 0 .
\end{gathered}
$$

Moreover,

$$
\int_{t}^{n-\tau(0)}\left\langle\left(\mathbf{1}_{\left[0, \zeta_{\tau}^{m}\right]}(r)-\mathbf{1}_{\left[0, \zeta_{\tau}^{n}\right]}(r)\right) \operatorname{sg} n\left(\delta Y_{r-}\right), \mathrm{d} A_{r}^{\mu}\right\rangle=0 .
$$

By what has been already proved, for every $t \geq 0$,

$$
\begin{aligned}
\left|\delta Y_{t}\right| \leq & E_{s, x}^{\prime}\left(\left|\varphi\left(\mathbf{X}_{n-s}\right)\right| \mathbf{1}_{\{\zeta>n-s\}}+\left|\varphi\left(\mathbf{X}_{m-s}\right)\right| \mathbf{1}_{\{\zeta>m-s\}}\right. \\
& \left.+\int_{n-s}^{\zeta} \mathrm{d}\left|A^{\mu}\right|_{r}+\int_{n-s}^{\zeta}\left|f\left(\mathbf{X}_{r}, 0\right)\right| \mathrm{d} r \mid \mathcal{F}_{t}^{\prime}\right) .
\end{aligned}
$$

The desired estimate now follows from Lemma 6.1 in [3] and Lemma 3.1. 


\subsection{Large-time behavior of solutions of PDEs}

In this subsection, we consider the operator $A$ defined by (4.10) (with coefficients not depending on time). Let us recall that for a nonnegative Borel measure $\mu$ on $D_{T}$ (respectively, $D$ ), we write

$R^{0, T} \mu(s, x)=\int_{D_{T}} p_{D}(s, x, t, y) \mathrm{d} \mu(t, y), \quad\left(\right.$ resp. $\left.R \mu(x)=\int_{D} G_{D}(x, y) \mathrm{d} \mu(y)\right)$.

LEMMA 6.2. Let $\mu \in \mathcal{M}_{0, b}^{+}\left(D_{T}\right)$. Then, there exists a PAF $A^{\mu}$ of $\mathbf{X}$ such that for every $(s, x) \in D_{T}$, if $R^{0, T} \mu(s, x)<\infty$, then

$$
E_{s, x}^{\prime} \int_{0}^{\zeta_{\tau}} \eta\left(\mathbf{X}_{t}\right) \mathrm{d} A_{t}^{\mu}=\int_{D_{T}} \eta(\theta, y) p_{D}(s, x, t, y) \mathrm{d} \mu(t, y)
$$

for every bounded $\eta \in \mathcal{B}\left(D_{T}\right)$.

Proof. See [13].

We say that a Borel measure $\mu$ on $D_{T}$ does not depend on time if for every $A \in$ $\mathcal{B}([0, T])$ and $B \in \mathcal{B}(D)$,

$$
\mu(A \times B)=\lambda(A) \cdot \tilde{\mu}(B)
$$

for some Borel measure $\tilde{\mu}$ on $D$ (Here, $\lambda$ stands for the Lebesgue measure). Since $\tilde{\mu}(B)=\mu([0,1] \times B)$ for $B \in \mathcal{B}(D), \tilde{\mu}$ is uniquely determined by $\mu$. From now on, given $\mu$ not depending on time, we will denote by $\tilde{\mu}$ the Borel measure on $D$ determined by (6.3). It is clear that if $\mu$ is soft with respect to cap, then $\tilde{\mu}$ is soft with respect to cap ${ }_{N}$ (see Sect. 4.3). It is known (see [9]) that there exists a unique continuous additive functional $A^{\tilde{\mu}}$ of $\mathbb{X}=\left\{\left(X, P_{x} \equiv P_{0, x}^{\prime}\right): x \in \mathbb{R}^{d}\right\}$ in the Revuz correspondence with measure $\tilde{\mu}$ or, equivalently, satisfying (4.12).

LEMMA 6.3. Let $\mu \in \mathcal{M}_{0, b}^{+}\left(D_{T}\right)$ do not depend on time. Then, $A^{\mu}$ is continuous, for every $s \in[0, T], R^{0, T} \mu(s, x)<\infty$ for q.e. $x \in D$ and

$$
A_{t}^{\mu}(0, \omega)=A_{t}^{\tilde{\mu}}(\omega) \text { for } P_{x} \text {-a.e. } \omega \in \Omega \text {. }
$$

Proof. Since $\left(X, P_{x}\right)$ is time homogeneous, $p_{D}(s, x, t, y)=p_{D}(t-s, x, y)$ for $t>$ $s, x, y \in D$. Taking into account that $\mu=\mathrm{d} t \otimes \tilde{\mu}$, we therefore have

$$
\begin{aligned}
\int_{D_{T}} p_{D}(s, x, \theta, y) \mathrm{d} \mu(\theta, y) & =\int_{0}^{T} \int_{D} p_{D}(\theta-s, x, y) \mathrm{d} \theta \tilde{\mu}(d y) \\
& \leq \int_{0}^{\infty} \int_{D} p_{D}(t, x, y) \tilde{\mu}(d y) \mathrm{d} t \\
& =E_{x} \int_{0}^{\zeta} \mathrm{d} A_{t}^{\tilde{\mu}}
\end{aligned}
$$


It is known (see [9]) that for every bounded smooth measure $\tilde{\mu}$ on $D$, the last integral above is finite for q.e. $x \in D$. This proves that for every $s \in[0, T], R^{0, T} \mu(s, x)$ $<\infty$ for q.e. $x \in D$. By [13], $E_{s, x}^{\prime}\left(A_{t}^{\mu}-A_{t-}^{\mu}\right)=\int_{D} p_{D}(s, x, t, y) \mu(t, d y)$, where $\mu(t, B)=\mu(\{t\} \times B)$ for $B \in \mathcal{B}(D)$. Since $\mu=\lambda \otimes \tilde{\mu}$, we conclude that $\mu(t, d y) \equiv 0$ for every $t \in[0, T]$, which implies that $A^{\mu}$ is continuous. We may assume that $\tilde{\mu} \in H^{-1}(D)$. If not, we argue as follows. It is known (see [9]) that for every smooth measure $\tilde{\mu}$ on $D$, there exists a generalized nest $\left\{F_{n}\right\}$ such that $\mathbf{1}_{F_{n}} \cdot \tilde{\mu} \in H^{-1}(D)$ for every $n \geq 1$. We prove the assertion for each measure $\mathbf{1}_{F_{n}} \cdot \tilde{\mu}$, and then, it is a simple matter to deduce the assertion for $\tilde{\mu}$. By the construction of the AF (see [13]), for every $(s, x) \in D_{T}$ such that $R^{0, T} \mu(s, x)<\infty$,

$$
E_{s, x}^{\prime} \sup _{0 \leq t \leq T_{\tau}}\left|A_{t}^{\mu_{n}}-A_{t}^{\mu}\right|^{2} \rightarrow 0,
$$

where $\mu_{n}=f_{n} \cdot m_{1}, f_{n}=n\left(u-u_{n}\right), A_{t}^{\mu_{n}}\left(\omega^{\prime}\right)=\int_{0}^{t} f_{n}\left(\mathbf{X}_{r}\left(\omega^{\prime}\right)\right) \mathrm{d} r, u$ is a solution to the problem

$$
\left\{\begin{array}{l}
\frac{\partial u}{\partial t}-A u=\mu, \\
u(0, \cdot)=\varphi, \quad u(t, \cdot)_{\mid \partial D}=0, t \in(0, T]
\end{array}\right.
$$

with some $\varphi \in L^{2}(D)$ and $u_{n}, n \in \mathbb{N}$, are solutions to the problems

$$
\left\{\begin{array}{l}
\frac{\partial u_{n}}{\partial t}-A u_{n}=n\left(u-u_{n}\right), \\
u_{n}(0, \cdot)=\varphi_{n}, \quad u_{n}(t, \cdot) \mid \partial D=0, t \in(0, T]
\end{array}\right.
$$

with $\varphi_{n} \in L^{2}(D)$ such that $\varphi_{n} \rightarrow u(0, \cdot)$ in $L^{2}(D)$. Since $\mu$ does not depend on time, one can choose $u \in H_{0}^{1}(D)$ such that $-A u=\tilde{\mu}$ and $u_{n} \in H_{0}^{1}(D)$ such that $-A u_{n}=n\left(u-u_{n}\right)$. By the construction of the PCAF $A^{\tilde{\mu}}$ of $\left(X, P_{x}\right)$,

$$
\int_{0}^{t} f_{n}\left(X_{r}(\omega)\right) \mathrm{d} r \rightarrow A_{t}^{\tilde{\mu}}, \quad t \geq 0
$$

for $P_{s, x}$-a.e. $\omega \in \Omega$. But the sequence $\left\{\int_{0}^{t} f_{n}\left(X_{r}(\omega)\right) \mathrm{d} r\right\}$ is convergent for $P_{x}$-a.e. $\omega \in \Omega$ iff $\left\{\int_{0}^{t} f_{n}\left(\mathbf{X}_{r}\left(\omega^{\prime}\right)\right) \mathrm{d} r\right\}$ is convergent for $P_{0, x}^{\prime}$-a.e. $\omega^{\prime} \in \Omega^{\prime}$, which in view of (6.4) gives the desired result.

From now on, we assume that the measure $\mu$ and the right-hand side $f$ do not depend on time. Let us consider the following parabolic system of PDEs

$$
\left\{\begin{array}{l}
\frac{\partial u^{k}}{\partial t}-A u^{k}=f^{k}(x, u)+\mu^{k}, \quad k=1, \ldots, N, \\
u_{\mid \partial D}(t, \cdot)=0, t \in(0, T],
\end{array}\right.
$$

and elliptic system of PDEs

$$
\left\{\begin{array}{l}
-A v^{k}=f^{k}(x, v)+\tilde{\mu}^{k} \quad \text { in } D, \quad k=1, \ldots, N, \\
v_{\mid \partial D}=0 .
\end{array}\right.
$$

Let $F_{0}=\{x \in D ;(0, x) \in F\}$, where $F$ is the set defined in Proposition 5.9 with $T=\infty($ see $(1.12))$. 
THEOREM 6.4. Assume (H1)-(H4) with $\alpha \leq 0$. Let $u, v$ be solutions of (6.5) and (6.6), respectively. Then, for every $t>0, q \in(0,1)$ and every $x \in F_{0}$,

$$
\begin{aligned}
|u(t, x)-v(x)| \leq & c(\Lambda)(1-q)^{-1 / q}\left(\left|P_{t} \varphi(x)\right| P_{x}^{(1-q) / q}\left(\zeta^{0}>t\right)+\left|\left(P_{t} R \tilde{\mu}\right)(x)\right|\right. \\
& \left.+\left|\left(P_{t} R f(\cdot, 0)\right)(x)\right|\right) .
\end{aligned}
$$

Proof. By Proposition 5.9, for every $x \in F_{0}$ (hence for q.e. $x \in D$ by Lemma 6.3), there exists a solution $\left(Y^{n}, Z^{n}\right)$ of $\operatorname{BDSE}_{0, x}$

$$
\begin{aligned}
Y_{t}^{n}= & \varphi\left(\mathbf{X}_{n-\tau(0)}\right) \mathbf{1}_{\{\zeta>n-\tau(0)\}}+\int_{t}^{\zeta_{\tau}^{n}} f\left(\mathbf{X}_{r}, Y_{r}^{n}\right) \mathrm{d} r \\
& +\int_{t}^{\zeta_{\tau}^{n}} \mathrm{~d} A_{r}^{\mu}-\int_{t}^{\zeta_{\tau}^{n}} Z_{r}^{n} \mathrm{~d} B_{r}, \quad 0 \leq t \leq \zeta_{\tau}^{n} .
\end{aligned}
$$

Let us recall that we put $Z_{t}^{n}=Y_{t}^{n}=0$ for $t \geq n-\tau(0)$. By Proposition 6.1, for every $n<m$ and $x \in F_{0}$, we have

$$
\begin{aligned}
& E_{0, x}^{\prime} \sup _{t \geq 0}\left|Y_{t}^{n}-Y_{t}^{m}\right|^{q}+E_{0, x}^{\prime}\left(\int_{0}^{\zeta}\left|Z_{r}^{n}-Z_{r}^{m}\right|^{2} \mathrm{~d} r\right)^{q / 2} \\
& \leq(1-q)^{-1}\left(1+2 C_{q}\right) E_{0, x}^{\prime}\left(\left|\varphi\left(\mathbf{X}_{n}\right)\right|^{q} \mathbf{1}_{\{\zeta>n\}}+\left|\varphi\left(\mathbf{X}_{m}\right)\right|^{q} \mathbf{1}_{\{\zeta>m\}}\right. \\
& \left.\quad+\left(\int_{n}^{\zeta} \mathrm{d}\left|A^{\mu}\right|_{r}\right)^{q}+\left(\int_{n}^{\zeta}\left|f\left(\mathbf{X}_{r}, 0\right)\right| \mathrm{d} r\right)^{q}\right)
\end{aligned}
$$

For every $t>0$,

$$
\begin{aligned}
E_{0, x}^{\prime}\left|\varphi\left(\mathbf{X}_{t}\right)\right|^{q} \mathbf{1}_{\{\zeta>t\}} & =E_{x}\left|\varphi\left(X_{t}\right)\right|^{q} \mathbf{1}_{\left\{\zeta^{0}>t\right\}} \\
& \leq\left(E_{x}\left|\varphi\left(X_{t}\right)\right|\right)^{q} \cdot P_{x}^{1-q}\left(\zeta^{0}>t\right) \\
& =\mid\left(\left.P_{t} \varphi(x)\right|^{q} \cdot P_{x}^{1-q}\left(\zeta^{0}>t\right)\right. \\
& \leq c(\Lambda) t^{-q d / 2}\|\varphi\|_{L^{1}}^{q} P_{x}^{1-q}\left(\zeta^{0}>t\right) .
\end{aligned}
$$

Also,

$$
\begin{aligned}
E_{0, x}^{\prime}\left(\int_{t}^{\zeta} \mathrm{d}\left|A^{\mu}\right|_{r}\right)^{q} & \leq\left(E_{0, x}^{\prime} \int_{t}^{\zeta} \mathrm{d}\left|A^{\mu}\right|_{r}\right)^{q}=\left(E_{x} \int_{t}^{\zeta^{0}} \mathrm{~d}\left|A^{\tilde{\mu}}\right|_{r}\right)^{q}=\left(E_{x} E_{X_{t}} \int_{0}^{\zeta^{0}} \mathrm{~d}\left|A^{\tilde{\mu}}\right|_{r}\right)^{q} \\
& =\left(E_{x}(R \tilde{\mu})\left(X_{t}\right)\right)^{q}=\left(P_{t}(R \tilde{\mu})(x)\right)^{q} \leq c(\Lambda) t^{-d q / 2}\|R \tilde{\mu}\|_{L^{1}}^{q} .
\end{aligned}
$$

Since $\|R \tilde{\mu}\|_{L^{1}}=\langle R \tilde{\mu}, 1\rangle_{L^{2}}$ and by (4.13), $(R 1)(x)=E_{x} \zeta^{0} \leq c(d, \Lambda)|D|^{d / 2}$, it follows that

$$
\|R \tilde{\mu}\|_{L^{1}} \leq c(d, \Lambda)|D|^{d / 2}\|\tilde{\mu}\|_{T V} .
$$

Similarly, since $\int_{0}^{t}\left|f\left(\mathbf{X}_{r}, 0\right)\right| \mathrm{d} r=\int_{0}^{t} \mathrm{~d}\left|A^{v}\right|_{r}, t \geq 0$, where $v=f(\cdot, 0) \cdot m$, we have

$$
E_{0, x}^{\prime}\left(\int_{t}^{\zeta}\left|f\left(\mathbf{X}_{r}, 0\right)\right| \mathrm{d} r\right)^{q} \leq c(d, \Lambda) t^{-d q / 2}\|R v\|_{L^{1}}^{q}
$$


and

$$
\|R v\|_{L^{1}} \leq c(d, \Lambda)|D|^{d / 2}\|f(\cdot, 0)\|_{L^{1}} .
$$

From (6.9)-(6.14), it follows that

$$
E_{0, x}^{\prime} \sup _{t \geq 0}\left|Y_{t}^{n}-Y_{t}^{m}\right|^{q}+E_{0, x}^{\prime}\left(\int_{0}^{\zeta}\left|Z_{r}^{n}-Z_{r}^{m}\right|^{2} \mathrm{~d} r\right)^{q / 2} \rightarrow 0
$$

as $n, m \rightarrow \infty$. Let us denote by $(Y, Z)$ the limit of the sequence $\left\{\left(Y^{n}, Z^{n}\right)\right\}$. It is clear from the above that $(Y, Z) \in S^{q} \otimes M^{q}$ for $q \in(0,1)$. For fixed $\varepsilon, R>0$, we have

$$
\begin{aligned}
P_{0, x}^{\prime} & \left(\int_{0}^{\zeta}\left|f\left(\mathbf{X}_{r}, Y_{r}^{n}\right)-f\left(\mathbf{X}_{r}, Y_{r}\right)\right| \mathrm{d} r>\varepsilon\right) \\
\leq & P_{0, x}^{\prime}\left(\int_{0}^{\zeta}\left|f\left(\mathbf{X}_{r}, Y_{r}^{n}\right)-f\left(\mathbf{X}_{r}, Y_{r}\right)\right| \mathrm{d} r>\varepsilon, \sup _{t \geq 0}\left|Y_{t}\right| \leq R, \sup _{t \geq 0}\left|Y_{t}^{n}-Y_{t}\right| \leq R\right) \\
& +P_{0, x}^{\prime}\left(\sup _{t \geq 0}\left|Y_{t}\right|>R\right)+P_{0, x}^{\prime}\left(\sup _{t \geq 0}\left|Y_{t}^{n}-Y_{t}\right|>R\right) \\
\equiv & I_{1}(n, R, \varepsilon)+I_{2}(R)+I_{3}(n, R) .
\end{aligned}
$$

By (H1), (H4), and the Lebesgue dominated convergence theorem, $I_{1}(n, R, \varepsilon) \rightarrow 0$ as $n \rightarrow \infty$. By (6.9), $I_{3}(n, R) \rightarrow 0$ as $n \rightarrow \infty$. Finally, since $Y \in S^{q}$ for $q \in$ $(0,1), I_{2}(R) \rightarrow 0$ as $R \rightarrow+\infty$. This shows that

$$
\int_{t}^{\zeta} f\left(\mathbf{X}_{r}, Y_{r}^{n}\right) \mathrm{d} r \rightarrow \int_{t}^{\zeta} f\left(\mathbf{X}_{r}, Y_{r}\right) \mathrm{d} r
$$

in probability $P_{0, x}^{\prime}$ uniformly on $[0, \zeta]$. By (6.10), (6.15), and (6.16), we may pass to the limit in (6.8). We then get

$$
Y_{t}=\int_{t}^{\zeta} f\left(\mathbf{X}_{r}, Y_{r}\right) \mathrm{d} r+\int_{t}^{\zeta} \mathrm{d} A_{r}^{\mu}-\int_{t}^{\zeta} Z_{r} \mathrm{~d} B_{r}, \quad 0 \leq t \leq \zeta, \quad P_{0, x}^{\prime} \text {-a.s. }
$$

By the Itô-Meyer formula,

$$
\begin{aligned}
\left|Y_{t}^{n}\right| & \leq E_{0, x}^{\prime}\left(\int_{t}^{\zeta} f\left(\mathbf{X}_{r}, Y_{r}^{n}\right) \cdot \operatorname{sgn}\left(Y_{r}^{n}\right)+\left|\varphi\left(\mathbf{X}_{n}\right)\right| \mathbf{1}_{\{\zeta>n\}} \mid \mathcal{F}_{t}^{\prime}\right) \\
& \leq E_{0, x}^{\prime}\left(\int_{t}^{\zeta}\left|f\left(\mathbf{X}_{r}, 0\right)\right| \mathrm{d} r \mid \mathcal{F}_{t}^{\prime}\right)+E_{0, x}^{\prime}\left(\left|\varphi\left(\mathbf{X}_{n}\right)\right| \mathbf{1}_{\{\zeta>n\}} \mid \mathcal{F}_{t}^{\prime}\right) \\
& =E_{x}\left(\int_{t}^{\zeta^{0}}\left|f\left(X_{r}, 0\right)\right| \mathrm{d} r \mid \mathcal{F}_{t}\right)+E_{x}\left(\left|\varphi\left(X_{n}\right)\right| \mathbf{1}_{\left\{\zeta^{0}>n\right\}} \mid \mathcal{F}_{t}\right) .
\end{aligned}
$$

But for $t<n$,

$$
E_{x}\left(\left|\varphi\left(X_{n}\right)\right| \mid \mathcal{F}_{t}\right)=E_{X_{t}}\left|\varphi\left(X_{n-t}\right)\right|=\left(P_{n-t}|\varphi|\right)\left(X_{t}\right) \leq c(\Lambda)(n-t)^{-d / 2}\|\varphi\|_{L^{1}} .
$$

Therefore, letting $n \rightarrow \infty$ in (6.18), we obtain

$$
\left|Y_{t}\right| \leq E_{x}\left(\int_{0}^{\zeta}\left|f\left(X_{r}, 0\right)\right| \mathrm{d} r \mid \mathcal{F}_{t}\right)
$$


From the above inequality, we conclude that $Y$ is of class (D). Set $\left(Y^{0, n}(\omega), Z^{0, n}(\omega)\right)=$ $\left(Y^{n}(0, \omega), Z^{n}(0, \omega)\right)$ and $\left(Y^{0}(\omega), Z^{0}(\omega)\right)=(Y(0, \omega), Z(0, \omega))$ for $w \in \Omega$. Then, from (6.15), (6.17) and the fact that

$$
E_{x} \sup _{t \geq 0}\left|Y_{t}^{0, n}-Y_{t}^{0}\right|^{q}+E_{x}\left(\int_{0}^{\zeta^{0}}\left|Z_{r}^{0, n}-Z_{r}^{0}\right|^{2} \mathrm{~d} r\right)^{q / 2} \rightarrow 0
$$

it follows that

$$
Y_{t}^{0}=\int_{t}^{\zeta^{0}} f\left(X_{r}, Y_{r}^{0}\right) \mathrm{d} r+\int_{t}^{\zeta^{0}} \mathrm{~d} A_{r}^{\tilde{\mu}}-\int_{t}^{\zeta^{0}} Z_{r}^{0} \mathrm{~d} B_{r}^{0}, \quad 0 \leq t \leq \zeta^{0}, \quad P_{x} \text {-a.s. }
$$

By what has already been proved, the pair $\left(Y^{0}, Z^{0}\right)$ has integrability properties under which the solution to $\operatorname{BSDE}_{x}(\zeta, f+\mathrm{d} \tilde{\mu})$ is unique (see [18]). Therefore, from [15], it follows that $\left(Y^{0}, Z^{0}\right)$ has the representation

$Y_{t}^{0}=v\left(X_{t}\right), \quad 0 \leq t \leq \zeta^{0}, \quad P_{x}$-a.s.,$\quad Z^{0}=\sigma \nabla v(X)$ on $\left[0, \zeta^{0}\right] \times \Omega, \quad \mathrm{d} t \otimes P_{x}$-a.e.

Moreover, by Theorem 5.12,

$Y_{t}^{n}=u_{n}\left(\mathbf{X}_{t}\right), 0 \leq t \leq \zeta_{\tau}^{n}, P_{0, x}^{\prime}$-a.s., $Z^{n}=\sigma \nabla u_{n}(\mathbf{X})$ on $\left[0, \zeta_{\tau}^{n}\right] \times \Omega^{\prime}, \mathrm{d} t \otimes P_{0, x}^{\prime}$-a.e.,

where $u_{n}$ is a solution of the system

$$
\left\{\begin{array}{l}
\frac{\partial u_{n}}{\partial t}+A u_{n}=-f\left(x, u_{n}\right)-\mu, \\
u_{n}(n, \cdot)=\varphi, \quad u_{n}(t, \cdot) \mid \partial D=0, \quad t \in[0, n)
\end{array}\right.
$$

Therefore, if we put $\zeta_{\tau}^{n, 0}(\omega)=\zeta_{\tau}^{n}(0, \omega)$, then

$$
Y_{t}^{0, n}=u_{n}\left(t, X_{t}\right), \quad 0 \leq t \leq \zeta_{\tau}^{n, 0}, P_{x} \text {-a.s. }
$$

and

$$
Z^{0, n}=\sigma \nabla u_{n}(\cdot, X) \quad \text { on }\left[0, \zeta_{\tau}^{n, 0}\right] \times \Omega, \mathrm{d} t \otimes P_{x} \text {-a.e. }
$$

It is an elementary check that

$$
u_{n}(t, x)=u(n-t, x), \quad t \in[0, n], \quad x \in D .
$$

Letting $m \rightarrow \infty$ in (6.9) and using (6.19), we obtain

$$
\begin{aligned}
E_{x} \sup _{t \geq 0}\left|Y_{t}^{0, n}-Y_{t}^{0}\right|^{q} \leq & (1-q)^{-1} E_{x}\left(\left|\varphi\left(X_{n}\right)\right|^{q} \mathbf{1}_{\left\{\zeta^{0}>n\right\}}+\left(\int_{n}^{\zeta^{0}} \mathrm{~d}\left|A^{\tilde{\mu}}\right|_{r}\right)^{q}\right. \\
& \left.+\left(\int_{n}^{\zeta^{0}}\left|f\left(X_{r}, 0\right)\right| \mathrm{d} r\right)^{q}\right) .
\end{aligned}
$$

From this and (6.10), (6.11), (6.20)-(6.23), we get (6.7).

COROLLARY 6.5. Assume (H1)-(H4) with $\alpha \leq 0$. Let $u$ and $v$ be solutions of (6.5) and (6.6), respectively. Then, (1.11) is satisfied for q.e. $x \in D$. In particular, for q.e. $x \in D, u(t, x) \rightarrow v(x)$ as $t \rightarrow \infty$.

Proof. Follows from (6.7), (6.10)-(6.12), and (4.14). 


\section{Acknowledgements}

\section{Research supported by Polish NCN Grant No. 2012/07/D/ST1/02107.}

Open Access. This article is distributed under the terms of the Creative Commons Attribution License which permits any use, distribution, and reproduction in any medium, provided the original author(s) and the source are credited.

\section{REFERENCES}

[1] D.G. Aronson, Non-Negative Solutions of Linear Parabolic Equations. Ann. Sc. Norm. Super. Pisa 22 (1968) 607-693.

[2] P. Bénilan, L. Boccardo, T. Gallouett, R. Gariepy, M. Pierre, J.- L. VazQuez, $L^{1}$-theory of existence and uniqueness of solutions of nonlinear elliptic equations. Ann. Scuola Norm. Sup. Pisa Cl. Sci. 22 (1995) 241-273.

[3] Ph. Briand, B. Delyon, Y. Hu, E. Pardoux, L. Stoica, $L^{p}$ solutions of Backward Stochastic Differential Equations. Stochastic Process. Appl. 108 (2003) 109-129.

[4] Ph. BRIAnd, R. CARMOnA, BSDEs with polynomial growth generators. J. Appl. Math. Stochastic Anal. 13 (2000) 207-238.

[5] K.L. Chung, Z. ZhaO, From Brownian Motion to Schrödinger's Equation. Springer, Berlin Heidelberg, 1995.

[6] J. Droniou, A. Porretta, A. Prignet, Parabolic Capacity and Soft Measures for Nonlinear Equations. Potential Anal. 19 (2003) 99-161.

[7] N. El Karoui, C. Kapoudjian, E. Pardoux, S. Peng, M.C. Quenez, Reflected solutions of backward SDEs, and related obstacle problems for PDE's. Ann. Probab. 25 (1997) 702-737.

[8] A. Friedman, Partial differential equations of parabolic type. Prentice-Hall, Englewood Cliffs, N.J., 1964.

[9] M. Fukushima, Y. Oshima, M. TAKedA, Dirichlet Forms and Symmetric Markov Processes. Walter de Gruyter, Berlin, 1994.

[10] S. Hamadène, M. HASSAni, BSDEs with two reflecting barriers: the general result. Probab. Theory Relat. Fields 132 (2005) 237-264.

[11] L.V. Kantorowich, B.Z. Vulih, A.G. Pinsker, Functional Analysis in Partially Ordered Spaces (in Russian). Gostekhizdat, Moscow, 1950.

[12] J. KISYNSKI, Convergence du type L. Colloq. Math. 7 (1960) 205-211.

[13] T. KLIMSIAK, Reflected BSDEs and the obstacle problem for semilinear PDEs in divergence form. Stochastic Process. Appl. 122 (2012) 134-169.

[14] T. KLIMSIAK, Cauchy problem for semilinear parabolic equation with time-dependent obstacles: a BSDEs approach. Potential Anal. 39 (2013) 99-140.

[15] T. Klimsiak, Semilinear elliptic systems with measure data. Ann. Mat. Pura Appl. (4) (2013) doi:10.1007/s10231-013-0364-4.

[16] T. KlimsiaK, On Time-Dependent Functionals of Diffusions Corresponding to Divergence Form Operators. J. Theoret. Probab. 26 (2013) 437-473

[17] T. KLImsiaK, Semi-Dirichlet forms, Feynman-Kac functionals and the Cauchy problem for semilinear parabolic equations (2014) arXiv:1401.3643v1

[18] T. KlimsiaK, A. RozKosz, Dirichlet forms and semilinear elliptic equations with measure data. J. Funct. Anal. 265 (2013) 890-925.

[19] T. Klimsiak, A. RozKosz, Obstacle problem for semilinear parabolic equations with measure data (2012). arXiv:1301.5795

[20] A. LEJAY, A probabilistic representation of the solution of some quasi-linear PDE with a divergence form operator. Application to existence of weak solutions of FBSDE, Stochastic Process. Appl. 110 (2004) 145-176.

[21] T. LeOnORI, F. PETITTA, Asymptotic behavior for solutions of parabolic equations with natural growth terms and irregular data. Asymptot. Anal. 48 (2006), 219-233. 
[22] P.- A. MEYER, Fonctionnelles multiplicatives et additives de Markov. Ann. Inst. Fourier 12 (1962) $125-230$.

[23] L. OrsinA, A.C. PONCE, Semilinear elliptic equations and systems with diffuse measures. J. Evol. Equ. 8 (2008) 781-812.

[24] Y. Oshima, On construction of Markov processes associated with time dependent Dirichlet spaces. Forum Math. 4 (1992) 395-415.

[25] Y. Oshima, Time-dependent Dirichlet forms and related stochastic calculus. Infin. Dimens. Anal. Quantum Probab. Relat. Top. 7 (2004) 281-316.

[26] E. PARDOUX, BSDEs, weak convergence and homogenization of semilinear PDEs, Nonlinear Analysis, Differential Equations and Control (Montreal, QC, 1998). Kluwer Academic Publishers, Dordrecht, 1999, pp. 503-549.

[27] F. PETITTA, Asymptotic behavior of solutions for linear parabolic equations with general measure data. C. R. Math. Acad. Sci. Paris 344 (2007) 571-576.

[28] F. PETITTA, Asymptotic behavior of solutions for parabolic operators of Leray-Lions type and measure data. Adv. Differential Equations 12 (2007) 867-891.

[29] F. PETITTA, Large time behavior for solutions of nonlinear parabolic problems with sign-changing measure data. Electron. J. Differential Equations (2008) No. 132, 10 pp.

[30] D. REvuZ, Mesures associees aux fonctionnelles additives de Markov I. Trans. Amer. Math. Soc. 148 (1970) 501-531.

[31] A. RozKOSZ, Weak convergence of diffusions corresponding to divergence form operators. Stochastics Stochastics Rep. 57 (1996) 129-157.

[32] A. RozKOSZ, Backward SDEs and Cauchy problem for semilinear equations in divergence form. Probab. Theory Related Fields 125 (2003) 393-407.

[33] L. SłominsKI, Stability of stochastic differential equations driven by general semimartingales. Dissertationes Math. 349 (1996) 113 pp.

[34] G. StAmPACCHIA, Équations elliptiques du second ordre à coefficients discontinus. Séminaire de Mathémtiques Supérieures 16 (1966) 326 pp.

\section{T. Klimsiak}

Faculty of Mathematics and

Computer Science,

Nicolaus Copernicus University,

Chopina 12/18, 87-100 Toruń,

Poland

E-mail: tomas@mat.umk.pl 